\title{
HOMOLOGY TORSION GROWTH AND MAHLER MEASURE
}

\author{
THANG LE
}

\begin{abstract}
We prove a conjecture of K. Schmidt in algebraic dynamical system theory on the growth of the number of components of fixed point sets. We also generalize a result of Silver and Williams on the growth of homology torsions of finite abelian covering of link complements. In both cases, the growth is expressed by the Mahler measure of the first non-zero Alexander polynomial of the corresponding modules. We use the notion of pseudo-isomorphism, and also tools from commutative algebra and algebraic geometry, to reduce the conjectures to the case of torsion modules. We also describe concrete sequences which give the expected values of the limits in both cases. For this part we utilize a result of Bombieri and Zannier (conjectured before by A. Schinzel) and a result of Lawton (conjectured before by D. Boyd).
\end{abstract}

\section{INTRODUCTION}

0.1. A conjecture of K. Schmidt. Suppose $M$ is a finitely generated module over the commutative ring $\mathfrak{R}:=\mathbb{Z}\left[t_{1}^{ \pm 1}, \ldots, t_{n}^{ \pm 1}\right]$. Let $\mathbb{S}$ be the unit circle in the complex plane $\mathbb{C}$. There is a natural action of $\mathbb{Z}^{n}$ on the compact abelian group $\hat{M}=\operatorname{Hom}(M, \mathbb{S})$, the Pontryagin dual of $M$. For details on dynamical systems of this type the reader is referred to the remarkable book $\mathrm{Sch}$. The entropy of this action, denoted by $h(M)$, can be defined in a standard manner. Lind, Schmidt, and Ward [LSW] (see also [EW]) proved that if $M$ is a torsion module, then

$$
h(M)=\mathbb{M}\left(\Delta_{0}(M)\right),
$$

where $\Delta_{0}(M)$ is the 0 -th Alexander polynomial of $M$ ( also known as the order of $\left.M\right)$, and $\mathbb{M}(f)$ is the additive Mahler measure of the polynomial $f$. We will recall the definitions of these notions in Section 1 .

For a subgroup $\Gamma \subset \mathbb{Z}^{n}$ of finite index let $\operatorname{Fix}_{\Gamma}(\hat{M})$ be the set of elements of $\hat{M}$ fixed by actions of elements of $\Gamma$. Then $\operatorname{Fix}_{\Gamma}(\hat{M})$ is a compact subgroup of $\hat{M}$ and has a finite number $P_{\Gamma}(\hat{M})$ of connected components. The following theorem was conjectured by K. Schmidt $\underline{\mathrm{Sch}}$, based on results in the torsion module case.

Theorem 1. For any finitely generated $\mathfrak{R}$-module $M$ one has

$$
\limsup _{\langle\Gamma\rangle \rightarrow \infty} \frac{\log P_{\Gamma}(\hat{M})}{\left|\mathbb{Z}^{n} / \Gamma\right|}=h(\operatorname{tor}(M)) .
$$

If $n=1$ then one can replace the limsup by the ordinary lim.

Here $\operatorname{tor}(M)$ is the torsion submodule of $M$, and

$$
\langle\Gamma\rangle=\min \{|x|, x \in \Gamma \backslash\{0\}\},
$$

where $|x|=\sqrt{\sum_{i}\left|x_{i}\right|^{2}}$ for $x=\left(x_{1}, \ldots, x_{n}\right) \in \mathbb{Z}^{n}$.

The theorem had been proved for the case when $M$ is a torsion module by Schmidt, see Sch, Theorem 21.1], and we will make substantial use of this case.

0.2. A conjecture of Silver and Williams. Suppose $L$ is an oriented link with $n$ ordered components in an oriented integral homology 3-sphere $Z$, with the complement $X=Z \backslash L$. There is a natural identification $H_{1}(X, \mathbb{Z})=\mathbb{Z}^{n}$. For a subgroup $\Gamma \subset \mathbb{Z}^{n}$ of finite index let $X_{\Gamma}$ be the corresponding abelian covering of $X$, and $X_{\Gamma}^{\mathrm{br}}$ the corresponding branched covering of $Z$. There are defined the Alexander polynomials $\Delta_{i}(L) \in \mathfrak{R}=\mathbb{Z}\left[t_{1}^{ \pm 1}, \ldots, t_{n}^{ \pm 1}\right], i=0,1,2, \ldots$ We will recall the definition of $\Delta_{i}(L)$ in section 4 .

The author was supported in part by National Science Foundation. Key words: torsion growth, Mahler measure, Alexander polynomials, algebraic dynamical system, entropy, pseudoisomorphism.

MSC: 57M10, 57M25, 57Q10, 37B50, 37B10. 
Let $\Delta(L)=\Delta_{j}(L)$, where $j$ is the smallest index such that $\Delta_{j}(L) \neq 0$. For an abelian group $G$, denote $\mathfrak{t o r}_{\mathbb{Z}}(G)$ the $\mathbb{Z}$-torsion subgroup of $G$.

Theorem 2. Notations as above. One has

$$
\begin{aligned}
& \limsup _{\langle\Gamma\rangle \rightarrow \infty} \frac{\log \left|\operatorname{tor}_{\mathbb{Z}}\left(H_{1}\left(X_{\Gamma}, \mathbb{Z}\right)\right)\right|}{\left|\mathbb{Z}^{n} / \Gamma\right|}=\mathbb{M}(\Delta(L)) . \\
& \limsup _{\langle\Gamma\rangle \rightarrow \infty} \frac{\log \left|\mathfrak{t o r}_{\mathbb{Z}}\left(H_{1}\left(X_{\Gamma}^{\mathrm{br}}, \mathbb{Z}\right)\right)\right|}{\left|\mathbb{Z}^{n} / \Gamma\right|}=\mathbb{M}(\Delta(L)) .
\end{aligned}
$$

If $n=1$ then one can replace the limsup by the ordinary lim.

For the special case when $\Delta(L)=\Delta_{0}(L)$, part (b) was proved by Silver and Williams SW, who, based on that result, formulated part (b), with the upper limit replaced by the ordinary limit, as a conjecture. The proof in [SW] (for the case $\Delta(L)=\Delta_{0}(L)$ ), written for $Z=S^{3}$ and for branched covering only, can be modified for the case of general homology 3-spheres and nonbranched coverings. Hence the real new content of Theorem 2 is the case when $\Delta(L) \neq \Delta_{0}(L)$. The proof in [SW] is based on the torsion module case of Theorem 1. It is not surprising that if one can get Theorem 1, then one can generalize the result of Silver and Williams to the case $\Delta(L) \neq \Delta_{0}(L)$.

The investigation of the growth of homology torsions of finite covering of knots has a long history, with an interesting conjecture posed by Gordon Go. The conjecture was proved by Riley Ri and Gonzalez-Acuna and Short GS using Gelfond-Baker results in number theory. Silver and Williams' result mentioned above and Theorem 2 are generalizations of Riley and GonzalezAcuna and H. Short from the knot to the link case. The surprising appearance of the Mahler measure can be explained from the perspective of $L^{2}$-torsion theory [Lü2]: The $L^{2}$-torsion of the maximal abelian covering of a link complement, at least when $\Delta_{0}(L) \neq 0$, is the Mahler measure of $\Delta_{0}(L)$. Theorem 2 more or less says that the $L^{2}$-torsion in this case can be approximated by its finite-dimensional counterparts.

In the non-abelian covering case the $L^{2}$-torsion is given by the hyperbolic volume. So one should expect the similar limit of the left hand side of Equation (a) would give the hyperbolic volume of the link complements. In Le2, we will show that, if $L$ is a non-split link in $S^{3}$, then

$$
\limsup _{\langle\Gamma\rangle \rightarrow \infty} \frac{\log \left|\operatorname{tor}_{\mathbb{Z}}\left(H_{1}\left(X_{\Gamma}, \mathbb{Z}\right)\right)\right|}{\left|\mathbb{Z}^{n} / \Gamma\right|} \leq \frac{\operatorname{vol}(L)}{6 \pi} .
$$

where $\operatorname{vol}(L)$ is the sum of the hyperbolic volumes of the hyperbolic pieces in the Jaco-ShalenJohansson decomposition of $S^{3} \backslash L$. Here $\Gamma$ runs the set of all subgroups of $\pi_{1}(L)$ of finite index, and $\langle\Gamma\rangle$ is the minimal word length of $\Gamma \backslash\{1\}$, measured using a fixed finite generator set of $\pi_{1}$. In particular, if $\operatorname{vol}(L)=0$, we have the equality in (2). For example, if $L$ is a torus knot, then one has equality in (2). For works in this direction see also [Le1, Mül, BV]. It is expected that the non-abelian case is much more complicated than the abelian case.

0.3. An algebraic version of Theorem 1, It is not difficult to reformulate Theorem 1 entirely in terms of module $M$, without going through the Pontryagin dual $\hat{M}$. We will show that Theorem 1 is equivalent to the following.

Theorem 3. For any finitely generated $\mathfrak{R}$-module $M$ one has

$$
\limsup _{\langle\Gamma\rangle \rightarrow \infty} \frac{\log \left|\mathfrak{t o r}_{\mathbb{Z}}\left(M \otimes \mathbb{Z}\left[\mathbb{Z}^{n} / \Gamma\right]\right)\right|}{\left|\mathbb{Z}^{n} / \Gamma\right|}=\mathbb{M}(\Delta(\operatorname{tor} M)) .
$$

If $n=1$ then one can replace the limsup by the ordinary lim.

Theorem 3 is a special case of the following.

Theorem 4. Suppose $\mathcal{C}$ is a chain complex of finitely generated free $\mathfrak{R}$-modules. Then for every $i \geq 0$,

$$
\limsup _{\langle\Gamma\rangle \rightarrow \infty} \frac{\log \left|\operatorname{tor}_{\mathbb{Z}}\left(H_{i}\left(\mathcal{C} \otimes \mathbb{Z}\left[\mathbb{Z}^{n} / \Gamma\right]\right)\right)\right|}{\left|\mathbb{Z}^{n} / \Gamma\right|}=\mathbb{M}\left(\Delta\left(H_{i}(\mathcal{C})\right)\right) .
$$

If $n=1$ then one can replace the limsup by the ordinary lim. 
In this paper we will prove Theorem 3 and from there deduce Theorem 2 and 4 ,

0.4. Application: abelian covering of $C W$-complex. Suppose $X$ is a finite $C W$-complex, equipped with a surjection $\rho: H_{1}(X, \mathbb{Z}) \rightarrow \mathbb{Z}^{n}$. Let $\tilde{X}$ be the abelian covering of $X$ corresponding $\rho$. The $C W$-structure of $X$ lifts to a $C W$-structure of $\tilde{X}$. The group $\mathbb{Z}^{n}$ acts as deck transformations on the covering $\tilde{X}$, making the cellular complex $\mathcal{C}(\tilde{X})$ of $\tilde{X}$ a free finitely-generated $\mathfrak{R}$-complex.

For every subgroup $\Gamma \in \mathbb{Z}_{n}$ of finite index let $\rho_{\Gamma}: \pi_{1}(X) \rightarrow A_{\Gamma}=\mathbb{Z}^{n} / \Gamma$ be the composition $\pi_{1}(X) \rightarrow H_{1}(X, \mathbb{Z}) \stackrel{\rho}{\longrightarrow} \mathbb{Z}^{n} \rightarrow A_{\Gamma}$, where the first map is the abelianization map. Let $X_{\Gamma}$ be the finite regular covering corresponding to $\rho_{\Gamma}$. Apply Theorem 4 to the complex $\mathcal{C}(\tilde{X})$ we get the following.

Theorem 5. Notations as above. Then

$$
\limsup _{\langle\Gamma\rangle \rightarrow \infty} \frac{\left|\mathfrak{t o r}_{\mathbb{Z}}\left(H_{1}\left(X_{\Gamma}, \mathbb{Z}\right)\right)\right|}{\left|\mathbb{Z}^{n} / \Gamma\right|}=\mathbb{M}\left(\Delta\left(\mathfrak{t o r}_{\mathfrak{R}} H_{i}(\tilde{X}, \mathbb{Z})\right)\right) .
$$

If $n=1$ then limsup can be replaced by the ordinary lim.

0.5. Ideas of Proofs. To prove Theorem 3 (and the equivalent Theorem11) we will reduce it to the case when $M$ is a torsion module, which had been proved in $[\mathrm{Sch}$, and the case when $M$ is torsionfree, i.e. when $\operatorname{tor}(M)=0$. Although the fact that $M$ is isomorphic to $\operatorname{tor}(M) \oplus(M / \operatorname{tor}(M))$ is not true in general, it would hold true if we replace isomorphism by pseudo-isomorphism, a notion introduced by Bourbaki Bou. The notion of pseudo-isomorphism is important for us, and we will review it in section 1 . The following will be one of the main technical results used in the proof of Theorem 3

Theorem 6. Suppose $M_{1}$ and $M_{2}$ are two pseudo-isomorphic finitely generated $\mathfrak{R}$-modules. Then $\left|\mathfrak{t o r}_{\mathbb{Z}}\left(M_{1} \otimes \mathbb{Z}\left[\mathbb{Z}^{n} / \Gamma\right]\right)\right|$ and $\left|\mathfrak{t o r}_{\mathbb{Z}}\left(M_{2} \otimes \mathbb{Z}\left[\mathbb{Z}^{n} / \Gamma\right]\right)\right|$ have the same growth rate in the sense that

$$
\lim _{\langle\Gamma\rangle \rightarrow \infty}\left(\frac{\log \left|\operatorname{tor}_{\mathbb{Z}}\left(M_{1} \otimes \mathbb{Z}\left[\mathbb{Z}^{n} / \Gamma\right]\right)\right|}{\left|\mathbb{Z}^{n} / \Gamma\right|}-\frac{\log \left|\mathfrak{t o r}_{\mathbb{Z}}\left(M_{2} \otimes \mathbb{Z}\left[\mathbb{Z}^{n} / \Gamma\right]\right)\right|}{\left|\mathbb{Z}^{n} / \Gamma\right|}\right)=0 .
$$

Note that the limit in Theorem 6 is the ordinary limit, not the upper limit.

In general, the direct calculation of $\left|\mathfrak{t o r}_{\mathbb{Z}}\left(M \otimes \mathbb{Z}\left[\mathbb{Z}^{n} / \Gamma\right]\right)\right|\left(\right.$ resp. the exact value or $\left.\left|\mathfrak{t o r}_{\mathbb{Z}}\left(H_{1}\left(X_{\Gamma}^{\mathrm{br}}, \mathbb{Z}\right)\right)\right|\right)$ is very difficult, especially in the case when $M$ is not a torsion module (resp. the 0-th Alexander polynomial is 0$)$. The only known formula for $\operatorname{tor}_{\mathbb{Z}}\left(X_{\Gamma}^{\mathrm{br}}, \mathbb{Z}\right)$, due to Mayberry and Murasugi $[\mathrm{MM}$, applies only to the case when $H_{1}\left(\left(X_{\Gamma}^{\mathrm{br}}, \mathbb{Z}\right)\right)$ itself is a torsion $\mathbb{Z}$-group. When the 0-th Alexander polynomial is 0 , which is the case concerned in this paper, the only known result is that of Hillman and Sakuma [HS] who calculated part of the torsion $\operatorname{tor}_{\mathbb{Z}}\left(H_{1}\left(X_{\Gamma}^{\mathrm{br}}, \mathbb{Z}\right)\right)$. The other not-yet-calculated part is related to the more difficult theory of modular representations of finite groups.

To circumvent this problem, we use an approximation $\beta(\Gamma)$ of $\mathbb{Z}\left[\mathbb{Z}^{n} / \Gamma\right]$, for which the calculation of $\left|\mathfrak{t o r}_{\mathbb{Z}}\left(M_{1} \otimes \beta\right)\right|$ is easier. Here $\beta(\Gamma)$ depends on $\Gamma$ and other data, and it approximates $\mathbb{Z}\left[\mathbb{Z}^{n} / \Gamma\right]$ in the sense that $\left|\operatorname{tor}_{\mathbb{Z}}\left(M_{1} \otimes \mathbb{Z}\left[\mathbb{Z}^{n} / \Gamma\right]\right)\right|$ and $\mid \mathfrak{t o r}_{\mathbb{Z}}\left(M_{1} \otimes \beta(\Gamma) \mid\right.$ have the same growth rate. The construction of $\beta(\Gamma)$ is based on the theory of torsion points on algebraic varieties. Needless to say, we have to use tools in commutative and homological algebra to get the desire estimates.

0.6. Sequence of converging subgroups. Theorem 3 guarantees there is a sequence of subgroups $\Gamma_{s} \subset \mathbb{Z}^{n}$ of finite index such that

$$
\lim _{s \rightarrow \infty} \frac{\log \left|\mathfrak{t o r}_{\mathbb{Z}}\left(M \otimes \mathbb{Z}\left[\mathbb{Z}^{n} / \Gamma_{s}\right]\right)\right|}{\left|\mathbb{Z}^{n} / \Gamma_{s}\right|}=\mathbb{M}(\Delta(\operatorname{tor} M)) .
$$

In the case when $M$ is a torsion module, half of the proof of Theorem $1 \mathrm{in}$ Sch is to construct such a sequence. The construction is long and difficult. In Section 5 (see Theorem 7 ) we give new sequences $\Gamma_{s}$ that work for both torsion and non-torsion modules. The proof is probably simpler, because we are able to use a result of Bombieri and Zannier [SZ, BMZ] on irreducibility of lacunary polynomials which was conjectured before by Schinzel, and a result of Lawton on approximation of Mahler measure which was a conjecture of Boyd. The methods and results of Section 5 are independent of the other parts and give an independent proof of "half" of Theorem 3 (or Theorem 1), namely that the left hand side of the identity of Theorem 3 is greater than or equal to the right hand side.

While writing this paper I was informed by Raimbault $\mathrm{Ra}$ that he gets an independent result similar to Theorem 7 of Section 5 by modifying the sequences in [Sch]. 
0.7. Acknowledgements. I would like to thank M. Baker, Hailong Dao, J. Hillman, K. Murasugi, S. Sakuma, K. Schmidt, D. Silver, and S. Williams for helpful conversations. I am particularly indebted to U. Zannier who has patiently explained to me over the course of 2 years many things from diophantine approximations to his (joint with Bombieri and Masser) remarkable work on anomalous subvariety structures, including a proof of a conjecture of Schinzel.

I lectured on parts of this work at conferences in Fukuoka (March 2009), Trieste (May 2009), Columbia University (June 2009), Osaka City University (November 2009), and would like to thank the organizers for the chance to give talks there.

The paper grew out of my attempt to prove a topological volume conjecture [Le1, Le2, This was part of a program aiming at understanding the question "Under what conditions $L^{2}$-torsions can be approximated by finite group counter parts?". I was attracted to this program while trying to develop an approach to attack the volume conjecture in quantum topology, and by the beautiful work of Lück work [Lü1] on approximation of $L^{2}$-Betti numbers.

0.8. Structure of the paper. Section 10 contains notations, basic facts (with some enhancements) about torsion points on algebraic varieties, pseudo-isomorphism, order of modules, lattices in Hermitian spaces, the integral group ring of finite abelian groups. It also contains a proof that Theorems 1 and 3 are equivalent. In Section 2, the main technical section, we present the construction the approximation $\beta$ of $\mathbb{Z}\left[\mathbb{Z}^{n} / \Gamma\right]$. Section 3 contains proofs of Theorems [6, 3, and 4 section प 4 gives a proof of Theorem 2 The last section contains the construction of converging sequences of lattices and Theorem 7

\section{Notations and Preliminaries}

1.1. Modules over $\mathfrak{R}=\mathbb{Z}\left[t_{1}^{ \pm 1}, \ldots, t_{n}^{ \pm 1}\right]$. Fix a free abelian group $\mathbb{Z}^{n}$. Let $\mathfrak{R}=\mathbb{Z}\left[\mathbb{Z}^{n}\right]$, which we identify with $\mathbb{Z}\left[t_{1}^{ \pm 1}, \ldots, t_{n}^{ \pm 1}\right]$ by sending $\mathbf{k}=\left(k_{1}, \ldots, k_{n}\right) \in \mathbb{Z}^{n}$ to $t^{\mathbf{k}}=\prod_{i=1}^{k} t_{i}^{k_{i}}$. The ring $\mathfrak{R}$ is a unique factorization Noetherian domain. In this paper $\mathfrak{R}$-modules are supposed to be finitely generated, and tensor products are assumed over $\mathfrak{R}$ unless otherwise indicated.

For a module $M$ over a commutative domain $R$, the torsion submodule $\operatorname{tor}_{R}(M)$ is defined by

$$
\operatorname{tor}_{R}(M)=\{x \in M \mid a x=0 \text { for some } 0 \neq a \in R\} .
$$

An $R$-module $M$ is a torsion module if $M=\operatorname{tor}_{R} M$. If $\operatorname{tor}_{R} M=0$, we call $M$ torsion-free. If $R=\mathfrak{R}$ we usually drop the subscript $\mathfrak{R}$ in the tor notation.

For a subgroup $\Gamma \subset \mathbb{Z}^{n}$ let $A_{\Gamma}:=\mathbb{Z} / \Gamma$ and $I(\Gamma)$ the ideal of $\mathbb{Z}\left[t_{1}^{ \pm 1}, \ldots, t_{n}^{ \pm 1}\right]$ generated by $\left\{1-t_{1}^{k_{1}} \ldots t_{n}^{k_{n}},\left(k_{1}, \ldots, k_{n}\right) \in \Gamma\right\}$. Then we have the following exact sequence

$$
0 \rightarrow I(\Gamma) \rightarrow \mathfrak{R} \stackrel{\mathrm{pr}}{\longrightarrow} \mathbb{Z}\left[A_{\Gamma}\right] \rightarrow 0 .
$$

Hence for every $\mathfrak{R}$-module $M$,

$$
M / I(\Gamma) M \cong M \otimes_{\mathfrak{R}} \mathbb{Z}\left[A_{\Gamma}\right] .
$$

Suppose $f, g$ are functions with positive real values on the set of subgroups $\Gamma \subset \mathbb{Z}^{n}$ of finite index. We say $f(\Gamma)$ has negligible growth rate if

$$
\lim _{\langle\Gamma\rangle \rightarrow \infty} f(\Gamma)^{1 /\left|\mathbb{Z}^{n} / \Gamma\right|}=1 .
$$

We say $f$ and $g$ have the same growth rate, and write $f \sim g$ if $f / g$ has negligible growth rate. Note that we do not require the individual $\operatorname{limit}_{\langle\Gamma\rangle \rightarrow \infty} f(\Gamma)^{1 /\left|\mathbb{Z}^{n} / \Gamma\right|}$ exists in this case.

We say two $\Re$-modules $M_{1}$ and $M_{2}$ have the same torsion growth, and write $M_{1} \sim M_{2}$, if

$$
\left|\mathfrak{t o r}_{\mathbb{Z}}\left(M_{1} \otimes \mathbb{Z}\left[A_{\Gamma}\right]\right)\right| \sim\left|\mathfrak{t o r}_{\mathbb{Z}}\left(M_{2} \otimes \mathbb{Z}\left[A_{\Gamma}\right]\right)\right| .
$$

1.2. Alexander polynomials. All definitions and facts here are standard and can be found in Tu, Hi].

Every finitely generated $\mathfrak{R}$-module $M$ has a presentation by an exact sequence

$$
\mathfrak{R}^{m_{1}} \stackrel{\partial_{1}}{\longrightarrow} \mathfrak{R}^{m_{0}} \rightarrow M \rightarrow 0,
$$

where $\partial_{1}$, given by a matrix of size $m_{1} \times m_{0}$ with entries in $\mathfrak{R}$, is known as a presentation matrix of $M$. A $k$-minor of $\partial_{1}$ is the determinant of any sub-matrix of size $k \times k$ of $\partial_{1}$. For $j \geq 0$, the $j$-th Alexander polynomial $\Delta_{j}(M)$ is the greatest common divisor of all the $\left(m_{0}-j\right)$-minor of $\partial_{1}$. 
It is known that $\Delta_{j}(M)$ depends only on $M$, but not on any particular presentation matrix. Each $\Delta_{j}(M)$ is defined up to units in $\mathfrak{R}$, so identity involving $\Delta_{j}(M)$ should be understood "up to units".

The 0-th polynomial $\Delta_{0}(M)$ is known as the order of $M$, which is non-zero if and only $M$ is a torsion module. Besides, $\Delta_{j}(M)$ divides $\Delta_{j-1}(M)$ for every $j \geq 1$.

The rank of a module $M$ over $\mathfrak{R}$ is the dimension of the vector space $M \otimes F(\mathfrak{R})$ over the fractional field $F(\mathfrak{R})$ of $\mathfrak{R}$. If $M$ has rank $r$, then $\Delta_{j}(M)=0$ if $j<r$, and

$$
\Delta_{j-r}(M)=\Delta_{j}(\operatorname{tor} M) .
$$

For any finitely-generated $\mathfrak{R}$-module of rank $r$, define

$$
\Delta(M):=\Delta_{r}(M)=\Delta_{0}(\operatorname{tor}(M))
$$

In case $M=\mathfrak{R} / I$, where $I=\left(f_{1}, \ldots, f_{l}\right)$ is the ideal generated by $f_{1}, \ldots, f_{l}$, then $\Delta_{0}(M)=$ $\operatorname{gcd}\left(f_{1}, \ldots, f_{l}\right)$, the greatest common divisor of the elements $f_{1}, \ldots, f_{l}$.

1.3. Pseudo-isomorphism. Reference for this part is $\mathrm{Bou}, \mathrm{Hi}$.

An $\mathfrak{R}$-module $N$ is pseudo-zero if for every prime ideal $\mathcal{P}$ of height 1 , the localization $N_{\mathcal{P}}$ is 0 . It is known that submodules and quotient modules of a pseudo-zero module are pseudo-zero.

In $\mathfrak{R}$, a prime ideal is of height 1 if and only if it is principal and generated by an irreducible polynomial.

An $\mathfrak{R}$-morphism $M_{1} \rightarrow M_{2}$ is a pseudo-isomorphism if the kernel and co-kernel are pseudo-zero.

Two finitely generated torsion $\Re$-modules $M_{1}, M_{2}$ are pseudo-isomorphic if and only $\Delta_{j}\left(M_{1}\right)=$ $\Delta_{j}\left(M_{2}\right)$ for every $j \geq 0$; in particular, a finitely generated torsion $\mathfrak{R}$-module is pseudo-zero if and only if $\Delta_{0}(M)=1$, see [Hi, Theorem 3.5].

Let us formulate some well-known facts in the form that will be useful for us.

Lemma 1.1. Suppose $I \subset \mathfrak{R}$ is a prime ideal, $I \neq \mathfrak{R}$.

a) $\mathfrak{R} / I$ is pseudo-zero if and only if $I$ is not principal.

b) If $\mathfrak{R} / I$ is pseudo-zero and $0 \neq p \in I$, then there is $q \in I$ such that $\operatorname{gcd}(p, q)=1$.

Proof. a) Since $I \neq \mathfrak{R}$ and $I$ is prime, $I=\left(p_{1}, \ldots, p_{l}\right)$, where $p_{i}$ 's are irreducible, non-unit, and distinct. One has

$$
\begin{aligned}
\mathfrak{R} / I \text { is pseudo-zero } & \Leftrightarrow \Delta_{0}(I)=\operatorname{gcd}\left(p_{1}, \ldots, p_{l}\right) \text { is } 1 \\
& \Leftrightarrow l \geq 2 \\
& \Leftrightarrow I \text { is not principal. }
\end{aligned}
$$

b) Suppose $q_{1}, \ldots, q_{l}$ are all irreducible factors of $p$. Suppose the contrary that every $q \in I$ is not co-prime with $p$, i.e. every $q \in I$ is divisible by one of $q_{i}$ 's. Then $I \subset \cup_{i=1}^{l}\left(q_{i}\right)$. Since each ideal $\left(q_{i}\right)$ is prime, there is an index $i$ such that $I \subset\left(q_{i}\right)$. Because $\left(q_{i}\right)$ has height 1 and $I$ is prime, this means $I=\left(p_{i}\right)$, which is principal. This contradicts the fact that $\mathfrak{R} / I$ is pseudo-zero.

The following is the main fact about pseudo-isomorphism which we will use.

Theorem 1.2. Bou, Theorem VII.4.5] Any finitely generated module $\mathfrak{R}$-module $M$ is pseudoisomorphic to $\operatorname{tor}(M) \oplus M / \operatorname{tor}(M)$.

Remark 1.1. It follows from (11) that if $M_{1}, M_{2}$ are pseudo-isomorphic, then they have the same entropy, $h\left(M_{1}\right)=h\left(M_{2}\right)$. In particular, if $M$ is pseudo-zero, then $h(M)=0$.

1.4. Equivalence of Theorem 1 and Theorem [3, Recall that $\mathbb{S}$ is the unit circle in $\mathbb{C}$. With the usual multiplication $\mathbb{S}$ is an abelian Lie group. For an abelian group $G$, the Pontryagin dual $\hat{G}=\operatorname{Hom}(G, \mathbb{S})$ is a compact group. If $G \cong \mathbb{Z}^{k}$, then $\hat{G} \cong \mathbb{S}^{k}$. On the other hand, if $|G|<\infty$, then $G \cong \hat{G}$. If $G \cong \mathbb{Z}^{k} \oplus \mathfrak{t o r}_{\mathbb{Z}}(G)$, then $\hat{G} \cong \mathbb{S}^{k} \times \widehat{\mathfrak{t o r}_{\mathbb{Z}}(G)}$. In particular, the cardinality $\left|\mathfrak{t o r}_{\mathbb{Z}} G\right|$ of the $\mathbb{Z}$-torsion $G$ is the number of connected components of the compact group $\hat{G}$. 
Suppose $M$ is a finitely generated $\mathfrak{R}$-module, and $\Gamma \subset \mathbb{Z}^{n}$ a subgroup of finite index. By definition

$$
\begin{aligned}
\operatorname{Fix}_{\Gamma}(\hat{M}) & =\{x \in \hat{M} \mid \gamma \cdot x=x \quad \forall \gamma \in \Gamma\} \\
& =\{x \in \hat{M}=\operatorname{Hom}(M, \mathbb{S}) \mid x(y)=x(\gamma(y)) \quad \forall \gamma \in \Gamma, y \in M\} \\
& =\{x \in \operatorname{Hom}(M, \mathbb{S}) \mid x((1-\gamma)(y))=1 \quad \forall \gamma \in \Gamma, y \in M\} \\
& =\{x \in \operatorname{Hom}(M, \mathbb{S}) \mid x(I(\Gamma) M)=1\} .
\end{aligned}
$$

It follows that

$$
\operatorname{Fix}_{\Gamma}(\hat{M}) \cong(M / I(\Gamma) M)^{\wedge} \cong\left(M \otimes \mathbb{Z}\left[A_{\Gamma}\right]\right)^{\wedge} .
$$

We can conclude that $P_{\Gamma}(\hat{M})$, the number of connected components of $\operatorname{Fix}_{\Gamma}(\hat{M})$, is

$$
P_{\Gamma}(\hat{M})=\left|\mathfrak{t o r}_{\mathbb{Z}}\left(M \otimes \mathbb{Z}\left[A_{\Gamma}\right]\right)\right| .
$$

From (11) and (3) we see that Theorem 1 and Theorem 3 are equivalent.

1.5. Theorem [3, the case when $M$ is a torsion module. As explained in Introduction, Theorem 3 in the case when $M$ is a torsion module, has been proved [Sch, Theorem 21.1]. We will use this result for the case $N$ is pseudo-zero. Since $\Delta_{0}(N)=1$ if $N$ is pseudo-zero, we have the following.

Proposition 1.3. Suppose $N$ is pseudo-zero. Then $N \sim 0$, i.e. $\left|\operatorname{tor}_{\mathbb{Z}}\left(N \otimes \mathbb{Z}\left[A_{\Gamma}\right]\right)\right| \sim 1$.

1.6. Lattices in Hermitian spaces and $\mathbb{Z}$-torsion. Suppose $W$ is a finite-dimensional based Hermitian space, i.e. a $\mathbb{C}$-vector space equipped with an Hermitian product (.,.) and a preferred orthonormal basis. The $\mathbb{Z}$-submodule $\Lambda \subset W$ spanned by the basis is called the fundamental lattice.

For a $\mathbb{Z}$-submodule (also called a lattice) $\Theta \subset \Lambda$ with $\mathbb{Z}$-basis $v_{1}, \ldots, v_{l}$ define

$$
\operatorname{vol}(\Theta)=\left|\operatorname{det}\left(\left(v_{i}, v_{j}\right)_{i, j=1}^{l}\right)\right|^{1 / 2} \text {. }
$$

It is clear that $\operatorname{vol}(\Theta) \geq 1$.

For a lattice $\Theta \subset \Lambda$ define its orthogonal complement in $\Lambda$ by

$$
\Theta^{\perp}=\{x \in \Lambda \mid(x, y)=0 \quad \forall y \in \Theta\} .
$$

It is clear that $\Theta \subset \Theta^{\perp \perp}$. A lattice $\Theta$ is primitive is $\Theta=\Theta^{\perp \perp}$. It is known that $\Theta$ is primitive if and only it is cut out by a subspace, i.e. $\Theta=\left(\Theta \otimes_{\mathbb{Z}} \mathbb{Q}\right) \cap \Lambda$; and if $\Theta$ is primitive, then (see e.g. Ber])

$$
\operatorname{vol}(\Theta)^{2}=\left|\Lambda /\left(\Theta \oplus \Theta^{\perp}\right)\right| .
$$

Lemma 1.4. For $i=1,2$ let $W_{i}$ be a finite-dimensional based Hermitian space with fundamental lattice $\Lambda_{i}$. Suppose $f: \Theta_{1} \rightarrow \Theta_{2}$ is a $\mathbb{Z}$-linear map, where $\Theta_{i} \subset \Lambda_{i}$ is a lattice of maximal rank. Then

$$
\left|\mathfrak{t o r}_{\mathbb{Z}}(\operatorname{coker} f)\right| \leq \|\left. f\right|^{\text {rk } \Theta_{2}} \operatorname{vol}\left(\Theta_{1}\right) .
$$

Here $\|f\|$ is the norm of the linear extension of $f$ to a $\mathbb{C}$-linear operator from $W_{1}$ to $W_{2}$.

Proof. Let $\overline{f\left(\Theta_{1}\right)}=\left(f\left(\Theta_{1}\right) \otimes_{\mathbb{Z}} \mathbb{Q}\right) \cap \Lambda_{2}$. Then

$$
\text { tor }_{\mathbb{Z}}(\operatorname{coker} f)=\overline{f\left(\Theta_{1}\right)} / f\left(\Theta_{1}\right) .
$$

Hence

$$
\begin{aligned}
\left|\mathfrak{t o r}_{\mathbb{Z}}(\operatorname{coker} f)\right| & =\left|\overline{f\left(\Theta_{1}\right)} / f\left(\Theta_{1}\right)\right| \\
& =\operatorname{vol}\left(f\left(\Theta_{1}\right) / \operatorname{vol}\left(\overline{f\left(\Theta_{1}\right)}\right) .\right.
\end{aligned}
$$

It is known that

$$
\operatorname{det}^{\prime}(f) \operatorname{vol}\left(\Theta_{1}\right)=\operatorname{vol}(\operatorname{ker} f) \operatorname{vol}\left(f\left(\Theta_{1}\right)\right),
$$

where $\operatorname{det}^{\prime}(f)$ is the product of all non-zero singular values of $f$, i.e. the square root of the product of all non-zero eigenvalues of $f^{*} f$. From (6) and (5) we have

$$
\left|\mathfrak{t o r}_{\mathbb{Z}}(\operatorname{coker} f)\right|=\frac{\operatorname{det}^{\prime}(f) \operatorname{vol}\left(\Theta_{1}\right)}{\operatorname{vol}(\operatorname{ker} f) \operatorname{vol}\left(\overline{f\left(\Theta_{1}\right)}\right)} \leq \operatorname{det}^{\prime}(f) \operatorname{vol}\left(\Theta_{1}\right) .
$$


The maximal singular value of $f$ is equal to $\|f\|$. The number of non-zero-singular values is less than or equal to the rank of $f$. Hence $\operatorname{det}^{\prime} f$, being the product of the non-zero singular value, is $\leq\|f\|^{\text {rk } L_{2}}$. Now from (7) we get the lemma.

\subsection{Decomposition of the group ring of a finite abelian group.}

1.7.1. Decomposition over $\mathbb{C}$. Suppose $A$ is a finite abelian group. The group $\operatorname{ring} \mathbb{C}[A]$ is a $\mathbb{C}$ vector space of dimension $|A|$. Equip $\mathbb{C}[A]$ with a Hermitian product so that $A$ is an orthonormal basis. Then the integral group ring $\mathbb{Z}[A]$ is the corresponding fundamental lattice.

The theory of representations of $A$ over $\mathbb{C}$ is easy: $\mathbb{C}[A]$ decomposes as a direct sum of mutually orthogonal one-dimensional $A$-modules:

$$
\mathbb{C}[A]=\bigoplus_{\chi \in \hat{A}} \mathbb{C} e_{\chi}
$$

where $e_{\chi}$ is the idempotent

$$
e_{\chi}=\frac{1}{|A|} \sum_{a \in A} \chi\left(a^{-1}\right) a .
$$

The vector subspaces $\mathbb{C} e_{\chi}$ 's are not only orthogonal with respect to the Hermitian structure, but also orthogonal with respect to the ring structure in the sense that $e_{\chi} e_{\chi^{\prime}}=0$ if $\chi \neq \chi^{\prime}$. Each $\mathbb{C} e_{\chi}$ is an ideal of the ring $\mathbb{Z}[A]$.

For a $\mathbb{Z}$-submodule $X \subset \mathbb{Z}[A]$ let $X_{\mathbb{C}}$ the $\mathbb{C}$-vector space spanned by $X$.

1.7.2. Decomposition corresponding to a subgroup. The integral group $\operatorname{ring} \mathbb{Z}[A]$ does not have as nice a decomposition as (8). Given a subgroup $B \subset A$, we decompose a subring of $\mathbb{Z}[A]$ as follows.

The natural projection $A \rightarrow A / B$ gives rise to the exact sequence

$$
0 \rightarrow \beta(B) \rightarrow \mathbb{Z}[A] \rightarrow \mathbb{Z}[A / B] \rightarrow 0,
$$

where $\beta(B)$ is the ideal of $\mathbb{Z}[A]$ generated by $1-b, b \in B$. As a lattice of $\mathbb{Z}[A], \beta(B)$ is primitive.

Let $\alpha(B)$ be the annihilator of $\beta(B)$ :

$$
\alpha(B)=\{x \in \mathbb{Z}[A] \mid x y=0 \quad \forall y \in \beta(B)\} .
$$

Then $\alpha(B)$ is also the orthogonal complement of $\beta(B)$ in $\mathbb{Z}[A]$. It is known that $\alpha(B)$ is the principal ideal generated by $u=u_{B}:=\sum_{b \in B} b$, see eg. [BM].

The complexification $\alpha_{\mathbb{C}}(B)$ and $\beta_{\mathbb{C}}(B)$ are easy to describe. Tensoring (9) with $\mathbb{C}$,

$$
0 \rightarrow \beta_{\mathbb{C}}(B) \rightarrow \mathbb{C}[A] \rightarrow \mathbb{C}[A / B] \rightarrow 0
$$

As a $\mathbb{C}[A]$-module, $\mathbb{C}[A / B]$ is isomorphic to $\alpha_{\mathbb{C}}(B)=\beta_{\mathbb{C}}(B)^{\perp}$, and

$$
\begin{gathered}
\alpha_{\mathbb{C}}(B)=\beta_{\mathbb{C}}(B)^{\perp}=\bigoplus_{\chi \in \hat{A},\left.\chi\right|_{B}=1} \mathbb{C} e_{\chi} \\
\operatorname{rk}_{\mathbb{Z}}(\alpha(B))=\operatorname{dim}_{\mathbb{C}}\left(\alpha_{\mathbb{C}}(B)\right)=|A| /|B| .
\end{gathered}
$$

Proposition 1.5. The finite group $\mathbb{Z}[A] /(\alpha(B) \oplus \beta(B))$ has order $|B|^{|A| /|B|}$.

Proof. Let $y_{1}, \ldots, y_{\ell} \in A$ be representatives of cosets of $B$ in $A$. Then $\ell=|A / B|$, and the elements $y_{1} u_{B}, \ldots, y_{\ell} u_{B}$ form a $\mathbb{Z}$-basis of $\alpha(B)$. It is easy to see that $\left(y_{i} u_{B}, y_{j} u_{B}\right)=0$ if $i \neq j$. The length of each vector $y_{j} u_{B}$ is $\left(\sum_{b \in B} \|\left. y_{i} b\right|^{2}\right)^{1 / 2}=|B|^{1 / 2}$. It follows that

$$
\operatorname{vol}(\alpha(B))=|B|^{\ell / 2} .
$$

From (41) we have $|\mathbb{Z}[A] /(\alpha(B) \oplus \beta(B))|=|B|^{\ell}=|B|^{|A / B|}$. 
1.7.3. Decomposition corresponding to a collection of subgroups. Suppose $B_{1}, \ldots, B_{k}$ are subgroups of a finite abelian group $A$. Let

$$
\alpha\left(B_{1}, \ldots, B_{k}\right)=\sum_{j=1}^{k} \alpha\left(B_{j}\right), \quad \beta\left(B_{1}, \ldots, B_{k}\right)=\bigcap_{j=1}^{k} \beta\left(B_{j}\right) .
$$

Then $\alpha\left(B_{1}, \ldots, B_{k}\right)$ and $\beta\left(B_{1}, \ldots, B_{k}\right)$ are primitive lattices of $\mathbb{Z}[A]$, and they are orthogonal complement of each other in $\mathbb{Z}[A]$. In addition, both $\alpha\left(B_{1}, \ldots, B_{k}\right)$ and $\beta\left(B_{1}, \ldots, B_{k}\right)$ are ideals of $\mathbb{Z}[A]$, and they are the annihilator of each other.

Proposition 1.6. The finite group $\mathbb{Z}[A] /\left(\beta\left(B_{1}, \ldots, B_{k}\right) \oplus \alpha\left(B_{1}, \ldots, B_{k}\right)\right)$ has order less than or equal to $\prod_{j=1}^{k}\left|B_{j}\right|^{|A| /\left|B_{j}\right|}$. Equivalently,

$$
\operatorname{vol}\left(\alpha\left(B_{1}, \ldots, B_{k}\right)\right) \leq\left(\prod_{j=1}^{k}\left|B_{j}\right|^{|A| /\left|B_{j}\right|}\right)^{1 / 2} .
$$

Proof. We write $\alpha=\alpha\left(B_{1}, \ldots, B_{k}\right)$ and $\beta=\beta\left(B_{1}, \ldots, B_{k}\right)$. Recall that $\beta=\cap_{j=1}^{k} \beta\left(B_{j}\right)$. We have

$$
\mathbb{Z}[A] /(\alpha+\beta) \cong(\mathbb{Z}[A] / \alpha) / \beta=(\mathbb{Z}[A] / \alpha) /\left(\cap_{j=1}^{k} \beta\left(B_{j}\right)\right) .
$$

Since $(\mathbb{Z}[A] / \alpha) /\left(\cap_{j=1}^{k} \beta\left(B_{j}\right)\right)$ injects in $\prod_{j=1}^{k}(\mathbb{Z}[A] / \alpha) / \beta\left(B_{j}\right)=\prod_{j=1}^{k}\left(\mathbb{Z}[A] / \beta\left(B_{j}\right)\right) / \alpha$, we have

$$
|\mathbb{Z}[A] /(\alpha+\beta)| \leq \prod_{j=1}^{k}\left|\left(\mathbb{Z}[A] / \beta\left(B_{j}\right)\right) / \alpha\right| .
$$

Since $\alpha\left(B_{j}\right) \subset \alpha,\left(\mathbb{Z}[A] / \beta\left(B_{j}\right)\right) / \alpha\left(B_{j}\right)$ surjects onto $\left(\mathbb{Z}[A] / \beta\left(B_{j}\right) / \alpha\right.$, hence

$$
\mid\left(\mathbb{Z}[A] / \beta\left(B_{j}\right) / \alpha|\leq|\left(\mathbb{Z}[A] / \beta\left(B_{j}\right)\right) / \alpha\left(B_{j}\right)|=| \mathbb{Z}[A] /\left(\alpha\left(B_{j}\right)+\beta\left(B_{j}\right)\right) \mid .\right.
$$

Inequalities (12) and (13), together with Proposition 1.5, show that

$$
|\mathbb{Z}[A] /(\alpha \oplus \beta)| \leq \prod_{j=1}^{k}\left|B_{j}\right|^{|A| /\left|B_{j}\right|} .
$$

The equivalence between the two statements follows from (44).

1.8. Torsion points in algebraic varieties. We recall well-known facts about algebraic subgroups of $\left(\mathbb{C}^{*}\right)^{n}$.

1.8.1. Algebraic subgroups of $\left(\mathbb{C}^{*}\right)^{n}$. With respect to the usual multiplication $\mathbb{C}^{*}:=\mathbb{C} \backslash\{0\}$ is an abelian group, and so is $\left(\mathbb{C}^{*}\right)^{n}$. An algebraic subgroup of $\left(\mathbb{C}^{*}\right)^{n}$ is a subgroup which is closed in the Zariski topology.

For a lattice, i.e. a subgroup, $\Lambda$ of $\mathbb{Z}^{n}$, not necessarily of maximal rank, recall that $I(\Lambda)$ is the ideal of $\mathfrak{R}$ generated by $1-t^{\mathbf{k}}, \mathbf{k} \in \Lambda$. Let $G(\Lambda)=V_{I(\Lambda)}$, the zero-set of $I(\Lambda)$, i.e. the set of all $\mathbf{z} \in \mathbb{C}^{n}$ such that $\mathbf{z}^{\mathbf{k}}-1=0$ for every $\mathbf{k} \in \Lambda$. Here for $\mathbf{k}=\left(k_{1}, \ldots, k_{n}\right) \in \mathbb{Z}^{n}$ and $\mathbf{z}=\left(z_{1}, \ldots, z_{n}\right) \in\left(\mathbb{C}^{*}\right)^{n}$ we set $t^{\mathbf{k}}=\prod_{i} t_{i}^{k_{i}}$ and $\mathbf{z}^{\mathbf{k}}=\prod_{i} z_{i}^{k_{i}}$.

It is easy to see that $G(\Lambda)$ is an algebraic subgroup. The converse holds true: Every algebraic subgroup is equal to $G(\Lambda)$ for some lattice $\Lambda$, see Sch-W.

Every element $\mathbf{z} \in G(\Lambda)$ defines a character $\chi_{\mathbf{z}}$ of the quotient group $A_{\Lambda}:=\mathbb{Z} / \Lambda$ via

$$
\chi_{\mathbf{z}}\left(t^{\mathbf{k}}\right)=\mathbf{z}^{\mathbf{k}},
$$

and conversely, every character of $A_{\Lambda}$ arises in this way. Thus one can identify $G(\Lambda)$ with $\operatorname{Hom}\left(A_{\Lambda}, \mathbb{C}^{*}\right)$ via $\mathbf{z} \rightarrow \chi_{\mathbf{z}}$. We will write $e_{\mathbf{z}}$ for the idempotent $\chi_{\chi_{\mathbf{z}}}$, and the decomposition (8), with $\Lambda$ having maximal rank, now becomes

$$
\mathbb{C}\left[A_{\Lambda}\right]=\bigoplus_{\mathbf{z} \in G(\Lambda)} \mathbb{C} e_{\mathbf{z}}
$$


1.8.2. Torsion points. A point $\mathbf{z} \in\left(\mathbb{C}^{*}\right)^{n}$ is a torsion point if it is a torsion element of the multiplicative group $\left(\mathbb{C}^{*}\right)^{n}$. Let $\mathbb{U}$ denote the set of all roots of unity in $\mathbb{C}^{*}$. Then the set of torsion points of $\left(\mathbb{C}^{*}\right)^{n}$ is $\mathbb{U}^{n}$. For example, if $\Gamma \subset \mathbb{Z}^{n}$ is a lattice of maximal rank, then $G(\Gamma) \subset \mathbb{U}^{n}$.

The following fact is well-known in the theory of torsion points on algebraic varieties.

Proposition 1.7. Suppose $X \neq \mathbb{C}^{n}$ is an algebraic subset of $\mathbb{C}^{n}$ defined over $\mathbb{Q}$. There exist a finite number of non-zero lattices $\Lambda_{1}, \ldots, \Lambda_{k}$ in $\mathbb{Z}^{n}$, such that $\mathbb{U}^{n} \cap X \subset \mathbb{U}^{n} \cap\left(\cup_{j=1}^{k} G\left(\Lambda_{j}\right)\right)$, i.e. any torsion point in $X$ belongs to $\cup_{j=1}^{k} G\left(\Lambda_{j}\right)$.

Proof. A torsion coset is a coset $u G$, where $u$ is torsion point and $G$ is an algebraic subgroup of $\left(\mathbb{C}^{*}\right)^{n}$. It is well known that there is a finite number of torsion cosets $u_{j} G_{j} \subset X$ such that every torsion point in $X$ belongs to $\cup_{j} u_{j} G_{j}$, see [Lau, Sch-W]. Since $u_{j} G_{j} \subset X$, the dimension of $G_{j}$ is at most $n-1$. Let $U_{j}$ be the finite cyclic group generated by $u_{j}$. Then $U_{j} G_{j}$ is also an algebraic group of dimension $\leq n-1$. Hence $U_{j} G_{j}=G\left(\Lambda_{j}\right)$, with $\Lambda_{j}$ a non-zero lattice. Since $u_{j} G_{j} \subset U_{j} G_{j}$, it is clear that every torsion point in $X$ belongs to $\cup_{j} G\left(\Lambda_{j}\right)$.

\subsection{Elementary bounds from exact sequences.}

Lemma 1.8. Suppose $M$ is a finitely-generated $\mathfrak{R}$-module with a free resolution

$$
\cdots \rightarrow \mathfrak{R}^{m_{2}} \stackrel{\partial_{2}}{\longrightarrow} \mathfrak{R}^{m_{1}} \stackrel{\partial_{1}}{\longrightarrow} \mathfrak{R}^{m_{0}} \rightarrow M \rightarrow 0,
$$

and $Q$ is a $\Re$-module with $|Q|<\infty$. Then $\left|\operatorname{Tor}_{i}^{\Re}(M, Q)\right| \leq|Q|^{m_{i}}$ for every $i=0,1, \ldots$

Proof. By definition, $\operatorname{Tor}_{i}^{\Re}(M, Q)$ is the homology groups of the complex

$$
\cdots \rightarrow \mathfrak{R}^{m_{2}} \otimes Q \rightarrow \mathfrak{R}^{m_{1}} \otimes Q \rightarrow \mathfrak{R}^{m_{0}} \otimes Q \rightarrow 0 .
$$

Since the $i$-th term of the this complex is $\mathfrak{R}^{m_{i}} \otimes Q \cong Q^{m_{i}}$, a finite group of order $|Q|^{m_{i}}$, its $i$-th homology group has $\leq|Q|^{m_{i}}$ elements.

Lemma 1.9. Suppose in an exact sequence of abelian groups

$$
\cdots \rightarrow N_{1}(\Gamma) \rightarrow M_{1}(\Gamma) \rightarrow M_{2}(\Gamma) \rightarrow N_{2}(\Gamma) \rightarrow \ldots
$$

each $M_{i}(\Gamma), N_{i}(\Gamma)$ is an abelian group depending on subgroups $\Gamma \subset \mathbb{Z}^{n}$ of finite index. Assume further that $N_{1}(\Gamma)$ and $N_{2}(\Gamma)$ are finite, and

$$
\left|N_{1}(\Gamma)\right| \sim 1 \sim\left|N_{2}(\Gamma)\right| .
$$

Then

$$
\left|\mathfrak{t o r}_{\mathbb{Z}}\left(M_{1}(\Gamma)\right)\right| \sim\left|\mathfrak{t o r}_{\mathbb{Z}}\left(M_{2}(\Gamma)\right)\right|
$$

Proof. Replacing $N_{1}$ by an appropriate quotient and $N_{2}$ by an appropriate subgroup, we may assume that

$$
0 \rightarrow N_{1}(\Gamma) \rightarrow M_{1}(\Gamma) \rightarrow M_{2}(\Gamma) \rightarrow N_{2}(\Gamma) \rightarrow 0
$$

is exact. We then have

$$
M_{1} / N_{1} \hookrightarrow M_{2} \rightarrow N_{2}
$$

The inclusion in (15) shows that

$$
\left|\mathfrak{t o r}_{\mathbb{Z}}\left(M_{1}\right)\right| /\left|N_{1}\right| \leq\left|\mathfrak{t o r}_{\mathbb{Z}}\left(M_{2}\right)\right|,
$$

and the surjecion in (15) shows that

$$
\mathfrak{t o r}_{\mathbb{Z}}\left(M_{2}\right) \leq\left|N_{2}\right|\left|\mathfrak{t o r}_{\mathbb{Z}}\left(M_{1} / N_{1}\right)\right|=\left|N_{2}\right|\left|\mathfrak{t o r}_{\mathbb{Z}}\left(M_{1}\right)\right| /\left|N_{1}\right| .
$$

From there we get the conclusion of the lemma.

Lemma 1.10. Suppose $\Theta$ is a free abelian group of finite rank, and $p, q: \Theta \rightarrow \Theta$ are $\mathbb{Z}$-linear operators with $p$ injective. Let $\mathcal{C}$ be the complex

$$
0 \rightarrow \Theta \stackrel{\partial_{2}}{\longrightarrow} \Theta^{2} \stackrel{\partial_{1}}{\longrightarrow} \Theta \rightarrow 0
$$

where $\partial_{2}(a)=(-q(a), p(a)), \partial_{1}(a, b)=p(a)+q(b)$. Then the homology groups of $\mathcal{C}$ are finite, and

$$
\left|H_{1}(\mathcal{C})\right|=\left|H_{0}(\mathcal{C})\right| \text {. }
$$


Proof. The complex $\mathcal{C}$ is the middle row of the commutative diagram with exact columns

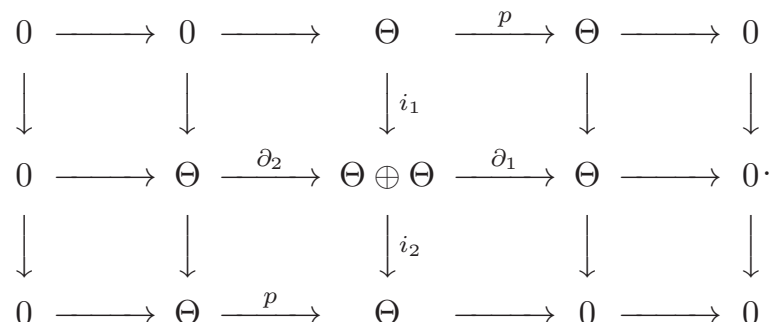

Here $i_{1}(a)=(a, 0), i_{2}(a, b)=b$. Let $\mathcal{C}_{1}$ be the first row and $\mathcal{C}_{2}$ the last row. Then $0 \rightarrow \mathcal{C}_{1} \rightarrow \mathcal{C} \rightarrow$ $\mathcal{C}_{2} \rightarrow 0$ is exact. The long exact sequence, together with $H_{1}\left(\mathcal{C}_{1}\right)=H_{0}\left(\mathcal{C}_{2}\right)$, gives us the following exact sequence

$$
0 \rightarrow H_{1}(\mathcal{C}) \rightarrow H_{1}\left(\mathcal{C}_{2}\right) \rightarrow H_{0}\left(\mathcal{C}_{1}\right) \rightarrow H_{0}(\mathcal{C}) \rightarrow 0 .
$$

Note that $\left|H_{1}\left(\mathcal{C}_{2}\right)\right|=\left|H_{0}\left(\mathcal{C}_{1}\right)\right|=\mid$ coker $p \mid$, which is finite since $p$ is injective. It follows that $H_{1}(\mathcal{C})$ and $H_{0}(\mathcal{C})$ are finite. In an exact sequence of finite abelian groups, the alternating product of the cardinalities is 1 . Hence, with the two middle terms having $\left|H_{1}\left(\mathcal{C}_{2}\right)\right|=\left|H_{0}\left(\mathcal{C}_{1}\right)\right|$, we must have $\left|H_{1}(\mathcal{C})\right|=\left|H_{0}(\mathcal{C})\right|$.

\section{Approximation of $\mathbb{Z}\left[\mathbb{Z}^{n} / \Gamma\right]$}

2.1. Approximation of $\mathbb{Z}\left[A_{\Gamma}\right]$ : Formulation of results. As mentioned in the introduction, we search for a good approximation of $\mathbb{Z}\left[\mathbb{Z}^{n} / \Gamma\right]$ as $\langle\Gamma\rangle \rightarrow \infty$. The approximation depends on some extra choice, namely, a non-zero element $p \in \mathfrak{R}$.

Fix a non-zero Laurent polynomial $p \in \mathfrak{R}=\mathbb{Z}\left[t^{ \pm 1}, \ldots, t^{ \pm n}\right]$. For each subgroup $\Gamma \subset \mathbb{Z}^{n}$ of rank $n$ we will construct an $\mathfrak{R}$-module $\beta(p ; \Gamma)$ with the following properties.

Proposition 2.1. (i) For every finitely generated $\mathfrak{R}$-module $M$ one has

$$
\left|\mathfrak{t o r}_{\mathbb{Z}}\left(M \otimes \mathbb{Z}\left[A_{\Gamma}\right]\right)\right| \sim\left|\mathfrak{t o r}_{\mathbb{Z}}(M \otimes \beta(p ; \Gamma))\right| .
$$

(ii) Suppose $p \in I$, where $I \neq \mathfrak{R}$ is ideal of $\mathfrak{R}$ such that $\mathfrak{R} / I$ is pseudo-zero. Then for each $i=0,1$ the module $\operatorname{Tor}_{i}^{\Re}(\beta(p ; \Gamma), \mathfrak{R} / I)$ is finite, and

$$
\left|\operatorname{Tor}_{i}^{\Re}(\beta(p ; \Gamma), \mathfrak{R} / I)\right| \sim 1 .
$$

The remaining part of this section is devoted to the construction of $\beta(p ; \Gamma)$ and the proof of Proposition 2.1

2.2. Heuristics. There is no rigorous mathematics in this subsection. Logically the reader can skip this subsection.

In the estimate of $\mathfrak{t o r}_{\mathbb{Z}}\left(M \otimes \mathbb{Z}\left[A_{\Gamma}\right]\right)$ using exact sequences, finiteness is very helpful. We will try to decompose $\mathbb{Z}[A]$ as a sum of two submodules, one is negligible, and the other if finite if tensoring with pre-given modules.

We have the decomposition (14) of $\mathbb{C}\left[A_{\Gamma}\right]$ into irreducible components

$$
\mathbb{C}\left[A_{\Gamma}\right]=\bigoplus_{\mathbf{z} \in G(\Gamma)} \mathbb{C} e_{\mathbf{z}}
$$

The module $M \otimes \mathbb{C}\left[A_{\Gamma}\right]$ will decompose accordingly. Albeit over $\mathbb{C}$, this gives us hint that some $\mathbf{z} \in G(\Gamma)$ are "good" and some are "bad". Here a good $\mathbf{z}$ must satisfy some non-degeneracy property, and if a point is good, all its Galois conjugates are good. Combining all good points together one should get some "integral" sub-module of $\mathbb{Z}\left[A_{\Gamma}\right]$ for which non-degeneracy conditions imply some kind of finiteness. If $S$ is the set of all bad points, and $S^{\perp}$ be its complement in $G(\Gamma)$, then one has

$$
\mathbb{C}\left[A_{\Gamma}\right]=\left(\bigoplus_{\mathbf{z} \in S} \mathbb{C} e_{\mathbf{z}}\right) \oplus\left(\bigoplus_{\mathbf{z} \in S^{\perp}} \mathbb{C} e_{\mathbf{z}}\right) .
$$

The module $\beta$ would be the "integral spine" of the second part.

The set of bad points will consists of those in $G(\Gamma)$ which are zeros $p$. For good points $\mathbf{z}$, $p(\mathbf{z}) \neq 0$, and this will give us the non-degeneracy condition. We control the set of bad points, which is the intersection $G(\Gamma) \cap V_{p}$, by using theory of torsion points on $V_{p}$, see Proposition 1.7 
2.3. Definition of $\beta(p ; \Gamma)$. The zero set

$$
V_{p}:=\left\{\left(z_{1}, \ldots, z_{n}\right) \in\left(\mathbb{C}^{*}\right)^{n} \mid p\left(z_{1}, \ldots, z_{n}\right)=0\right\}
$$

is an algebraic subset of $\left(\mathbb{C}^{*}\right)^{n}$ of dimension $\leq n-1$. Let $\Lambda_{1}, \ldots, \Lambda_{k}$ be the non-zero subgroups of $\mathbb{Z}^{n}$ described in Proposition 1.7 with $X=V_{p}$. By construction,

$$
\text { if a torsion point } \mathbf{z} \text { does not belong to } \bigcup_{j=1}^{k} G\left(\Lambda_{j}\right) \text {, then } p(\mathbf{z}) \neq 0 \text {. }
$$

Suppose $A=A_{\Gamma}:=\mathbb{Z}^{n} / \Gamma$, where $\Gamma \subset \mathbb{Z}^{n}$ is a subgroup of maximal rank $n$. The abelian groups $B_{j}=\left(\Lambda_{j}+\Gamma\right) / \Gamma$ are subgroups of $A=\mathbb{Z}^{n} / \Gamma$. Let

$$
\alpha(p ; \Gamma)=\alpha\left(B_{1}, \ldots, B_{k}\right), \quad \beta(p ; \Gamma)=\beta\left(B_{1}, \ldots, B_{k}\right),
$$

where $\alpha\left(B_{1}, \ldots, B_{k}\right)$ and $\beta\left(B_{1}, \ldots, B_{k}\right)$ are ideals of $\mathbb{Z}[A]$ defined as in Section 1.7.3.

We partition $G(\Gamma)=\hat{A}$ into two disjoint subsets $S, S^{\perp}$ by

$$
S=G(\Gamma) \bigcap\left(\bigcup_{j} G\left(\Lambda_{j}\right)\right), \quad S^{\perp}=G(\Gamma) \backslash S .
$$

We will see that as $\langle\Gamma\rangle \rightarrow \infty, S$ is small compared to its complement $S^{\perp}$. Note that $\chi_{\mathbf{z}}$, with $\mathbf{z} \in G(\Gamma)$, takes value 1 on $B_{j}$ exactly when $\mathbf{z} \in G\left(\Lambda_{j}\right)$. Hence from (10) we have

$$
\alpha_{\mathbb{C}}\left(B_{j}\right)=\bigoplus_{\mathbf{z} \in G(\Gamma) \cap G\left(\Lambda_{j}\right)} \mathbb{C}_{\mathbf{z}}
$$

and hence

$$
\beta_{\mathbb{C}}(p ; \Gamma)=\bigoplus_{\mathbf{z} \in S^{\perp}} \mathbb{C}_{\mathbf{z}}, \quad \alpha_{\mathbb{C}}(p ; \Gamma)=\bigoplus_{\mathbf{z} \in S} \mathbb{C}_{\mathbf{z}}
$$

We will write $\alpha=\alpha(p ; \Gamma), \beta=\beta(p ; \Gamma)$. Let $\operatorname{pr}: \mathfrak{R} \rightarrow \mathbb{Z}\left[A_{\Gamma}\right]$ be the canonical projection. Note that $\mathrm{pr}^{-1}(0)$ is the ideal of all polynomials taking values 0 at every point of $G(\Gamma)$. Similarly, $\tilde{\alpha}=\operatorname{pr}^{-1}(\alpha)$ is the ideal of all polynomials taking values 0 at every point of $S^{\perp}$. Over $\mathbb{C}, \tilde{\alpha}_{\mathbb{C}}$ is the reduced ideal of $\mathfrak{R}_{\mathbb{C}}=\mathbb{C}\left[t_{1}^{ \pm 1}, \ldots, t_{n}^{ \pm 1}\right]$ whose zero set is $S^{\perp}, V_{\tilde{\alpha}_{\mathbb{C}}}=S^{\perp}$. In addition,

$$
\mathfrak{R}_{\mathbb{C}} / \tilde{\alpha}_{\mathbb{C}} \cong \mathbb{C}[A] / \alpha_{\mathbb{C}} \cong \beta_{\mathbb{C}}
$$

The important facts concerning $\alpha(p ; \Gamma)$ and $\beta(p ; \Gamma)$ are the following.

Lemma 2.2. a) $S^{\perp}=V_{\tilde{\alpha}_{\mathbb{C}}}$ does not intersect $V_{p}$. It follows that the ideal of $\mathfrak{R}_{\mathbb{C}}$ generated by $p$ and $\tilde{\alpha}_{\mathbb{C}}$ is the whole $\mathfrak{R}_{\mathbb{C}}$.

b) The multiplication map $p: \beta \rightarrow \beta, x \rightarrow p \cdot x$, is injective. It follows that $\operatorname{Tor}_{1}^{\Re}(\Re /(p), \beta)=0$.

c) The quotient group $Q(p ; \Gamma):=\mathbb{Z}\left[A_{\Gamma}\right] /(\alpha(p ; \Gamma) \oplus \beta(p ; \Gamma))$ is finite and its order is negligible, $|Q(p ; \Gamma)| \sim 1$.

d) $|S|=\operatorname{rk}_{\mathbb{Z}} \alpha(p ; \Gamma)$ is small compared to the $\operatorname{rk}_{\mathbb{Z}} \mathbb{Z}\left[A_{\Gamma}\right]=\left|A_{\Gamma}\right|$ in the sense that

$$
\lim _{\langle\Gamma\rangle \rightarrow \infty} \frac{\operatorname{rk}_{\mathbb{Z}} \alpha(p ; \Gamma)}{\left|A_{\Gamma}\right|}=0 .
$$

e) One has $\operatorname{vol}(\alpha) \sim 1$.

Proof. a) Suppose $\mathbf{z} \in S^{\perp}$. By definition (17), $\mathbf{z}$ is a torsion point not belonging to $\bigcup_{j=1}^{k} G\left(\Lambda_{j}\right)$. By (16), $p(\mathbf{z}) \neq 0$. In other words, $V_{p} \cap V_{\tilde{\alpha}_{\mathbb{C}}}=\emptyset$. By Nullstellensatz, the ideal generated by $p$ and $\tilde{\alpha}_{\mathbb{C}}$ is the whole $\mathfrak{R}_{\mathbb{C}}$.

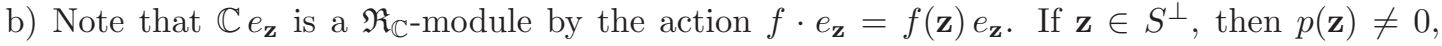
hence $p: \mathbb{C} e_{\mathbf{z}} \rightarrow \mathbb{C} e_{\mathbf{z}}$ is an isomorphism. Since $\beta_{\mathbb{C}}=\bigoplus_{\mathbf{z} \in S^{\perp}} \mathbb{C} e_{\mathbf{z}}$, the map $p: \beta_{\mathbb{C}} \rightarrow \beta_{\mathbb{C}}$ is also an isomorphism. It follows that $p: \beta \rightarrow \beta$ is injective.

One has $\operatorname{Tor}_{1}^{\Re}(\Re /(p), \beta)=\operatorname{ker}(p: \beta \rightarrow \beta, x \rightarrow p \cdot x)=0$.

c) We will first show that for each $j=1, \ldots, k$,

$$
\lim _{\langle\Gamma\rangle \rightarrow \infty}\left|B_{j}\right|=\infty .
$$


By definition, $B_{j}=\left(\Lambda_{j}+\Gamma\right) / \Gamma$. Fix an element $x \in \Lambda_{j}, x \neq 0$, and look at the degree of $x$ in $B_{j}=\left(\Lambda_{j}+\Gamma\right) / \Gamma$. If $m|x|<\langle\Gamma\rangle$, then $m|x|$ does not belong to $\Gamma$ by the definition of $\langle\Gamma\rangle$, and hence $m x$ is not 0 in $B_{j}=\left(\Lambda_{j}+\Gamma\right) / \Gamma$. This means the cyclic subgroup of $B_{j}$ generated by $x$ has order at least $\langle\Gamma\rangle /|x|$. It follows that $\left|B_{j}\right| \geq\langle\Gamma\rangle /|x|$. Hence $\lim _{\langle\Gamma\rangle \rightarrow \infty}\left|B_{j}\right|=\infty$.

From Proposition 1.6 .

$$
|Q(p ; \Gamma)|^{1 /|A|} \leq \prod_{j=1}^{k}\left|B_{j}\right|^{1 /\left|B_{j}\right|},
$$

from which and (20) we get $|Q(p ; \Gamma)| \sim 1$.

d) By (11) one has $\operatorname{rk}\left(\alpha\left(B_{j}\right)\right)=|A| /\left|B_{j}\right|$. Since $\alpha=\sum \alpha\left(B_{j}\right)$, one gets

$$
\operatorname{rk}(\alpha) /|A| \leq \sum_{j=1}^{k} \operatorname{rk} \alpha\left(B_{j}\right) /|A|=\sum_{j=1}^{k}\left(1 /\left|B_{j}\right|\right),
$$

which, with (20), shows that $\lim _{\langle\Gamma\rangle \rightarrow \infty} \frac{\mathrm{rk}_{\mathbb{Z}} \alpha(p ; \Gamma)}{\left|A_{\Gamma}\right|}=0$.

e) This follows immediately from (44) and part (c).

2.4. Contribution from $\alpha(p ; \Gamma)$ is negligible. The ideals $\alpha$ and $\beta$, being $\mathbb{Z}\left[A_{\Gamma}\right]$-module, can be naturally considered as $\mathfrak{R}$-modules.

Lemma 2.3. Suppose $M$ is a finitely generated $\mathfrak{R}$-module. Then

$$
\left|\mathfrak{t o r}_{\mathbb{Z}}(M \otimes \alpha(p ; \Gamma))\right| \sim 1 .
$$

Proof. Tensoring the presentation

$$
\mathfrak{R}^{m_{1}} \stackrel{\partial_{1}}{\longrightarrow} \mathfrak{R}^{m_{0}} \rightarrow M \rightarrow 0 .
$$

with $\mathbb{Z}[A]$ and $\alpha$ respectively, one gets

$$
\begin{gathered}
\left(\mathbb{Z}\left[A_{\Gamma}\right]\right)^{m_{1}} \stackrel{\partial_{1, \Gamma}}{\longrightarrow}\left(\mathbb{Z}\left[A_{\Gamma}\right]\right)^{m_{0}} \rightarrow M \otimes \mathbb{Z}\left[A_{\Gamma}\right] \rightarrow 0, \\
\alpha^{m_{1}} \stackrel{\partial_{1, \alpha}}{\longrightarrow} \alpha^{m_{0}} \rightarrow M \otimes \alpha \rightarrow 0,
\end{gathered}
$$

with $\partial_{1, \alpha}$ the restriction of $\partial_{1, \Gamma}$.

Recall that we have a Hermitian structure on $\mathbb{C}[A]$. It is not difficult to find an upper bound, not depending on $\Gamma$, for all the operator $\partial_{1, \Gamma}$. In fact, by [Lü1, Lemma 2.5],

$$
\left\|\mathcal{O}_{\Gamma}\right\| \leq D:=m_{1} m_{0} \max _{i, j}\left\{\left|\mathcal{O}_{i j}\right|_{1}\right\}
$$

where for a Laurent polynomial $a \in \mathbb{Z}\left[t_{1}^{ \pm 1}, \ldots, t_{n}^{ \pm 1}\right]$ the norm $|a|_{1}$ is the sum of the absolute values of its coefficients.

Because $\alpha_{\mathbb{C}}$ is an invariant subspace $\mathbb{C}[A]$, we also have

$$
\left\|\mathcal{O}_{\alpha}\right\| \leq D
$$

Applying Lemma 1.4 to the sequence (22) we get

$$
\left|\mathfrak{t o r}_{\mathbb{Z}}(M \otimes \alpha)\right| \leq D^{\mathrm{rk}_{\mathbb{Z}} \alpha} \operatorname{vol}(\alpha) .
$$

The right hand side has negligible growth, by Lemma 2.2(d) and (e).

\subsection{Proof of Proposition 2.1 part (i).}

Proof. Recall that $A=A_{\Gamma}:=\mathbb{Z}^{n} / \Gamma$. We have an exact sequence

$$
0 \rightarrow(\alpha \oplus \beta) \rightarrow \mathbb{Z}[A] \rightarrow Q \rightarrow 0,
$$

with $Q=|Q(p ; \Gamma)| \sim 1$ by Lemma 2.2 a. Tensoring (23) with $M$,

$$
\cdots \rightarrow \operatorname{Tor}_{1}^{\Re}(M, Q) \rightarrow((M \otimes \alpha) \oplus(M \otimes \beta)) \rightarrow M \otimes \mathbb{Z}[A] \rightarrow \operatorname{Tor}_{0}^{\Re}(M, Q) \rightarrow 0 .
$$

Lemma 1.8 shows that $\left|\operatorname{Tor}_{i}^{\Re}(M, Q)\right|<|Q|^{m_{i}}$ for some constant $m_{i}$ depending on $M$ only. Since $Q \sim 1$, we also have

$$
\left|\operatorname{Tor}_{i}^{\Re}(M, Q)\right| \sim 1 .
$$

Applying Lemma 1.9 to the sequence (24), we get 


$$
\left|\mathfrak{t o r}_{\mathbb{Z}}(M \otimes \alpha) \oplus \mathfrak{t o r}_{\mathbb{Z}}(M \otimes \beta)\right| \sim\left|\mathfrak{t o r}_{\mathbb{Z}}(M \otimes \mathbb{Z}[A])\right| .
$$

Since $\left|\mathfrak{t o r}_{\mathbb{Z}}(M \otimes \alpha)\right| \sim 1$ by Lemma 2.3 we have $\left|\mathfrak{t o r}_{\mathbb{Z}}(M \otimes \beta)\right| \sim\left|\mathfrak{t o r}_{\mathbb{Z}}(M \otimes \mathbb{Z}[A])\right|$.

2.6. The intermediate ideal $J=(p, q)$. To prepare for the proof of Proposition part (ii), we first study the ideal $J=(p, q)$, where $q \in \mathfrak{R}$ is co-prime with $p$. The reason is $\mathfrak{R} / J$ has a simple free resolution, and hence $\operatorname{Tor}_{i}^{\mathfrak{R}}(\mathfrak{R} / J, \beta(p ; \Gamma))$ is easy to study.

Lemma 2.4. Both modules $\operatorname{Tor}_{1}^{\Re}(\Re / J, \beta(p ; \Gamma))$ and $\operatorname{Tor}_{0}^{\Re \mathfrak{R}}(\mathfrak{R} / J, \beta(p ; \Gamma))$ are finite, have the same cardinality, and have negligible growth, i.e.

$$
\left|\operatorname{Tor}_{1}^{\Re}(\mathfrak{R} / J, \beta(p ; \Gamma))\right|=\left|\operatorname{Tor}_{0}^{\Re}(\Re / J, \beta(p ; \Gamma))\right| \sim 1 .
$$

Proof. We have the following free resolution of $\mathfrak{R} / J$

$$
0 \rightarrow \mathfrak{R} \stackrel{d_{2}}{\longrightarrow} \mathfrak{R} \oplus \mathfrak{R} \stackrel{d_{1}}{\longrightarrow} \mathfrak{R} \rightarrow \mathfrak{R} / J \rightarrow 0,
$$

where $d_{2}(a)=(-q a, p a)$ and $d_{1}(a, b)=p a+q b$. This can be directly checked easily, or can be deduced from the theory of Koszul complex as follows. Since $p, q$ are co-prime, the sequence $(p, q)$ is a regular sequence of $\mathfrak{R}$ (see Exercise 5 of page 102 of [Ka]). Hence the Koszul complex of $(p, q)$, which is (26), is a free resolution of $\mathfrak{R} / J$.

From the free resolution (26) $\operatorname{Tor}_{i}^{\mathfrak{R}}(\mathfrak{R} / J, \beta)$ is the $i$-th homology of the complex

$$
0 \rightarrow \beta \stackrel{(-q, p)}{\longrightarrow} \beta \oplus \beta \stackrel{\left(\begin{array}{l}
p \\
q
\end{array}\right)}{\longrightarrow} \beta \rightarrow 0 .
$$

The module $\beta$ is a free $\mathbb{Z}$-module of finite rank, and the map $p: \beta \rightarrow \beta$ is injective, by Lemma 2.2 (b). From Lemma 1.10 we see that both $\operatorname{Tor}_{1}^{\mathfrak{R}}(\mathfrak{R} / J, \beta)$ and $\operatorname{Tor}_{0}^{\mathfrak{R}}(\mathfrak{R} / J, \beta)=(\mathfrak{R} / J) \otimes \beta$ are finite, and

$$
\left|\operatorname{Tor}_{1}^{\Re}(\Re / J, \beta)\right|=|\Re / J \otimes \beta| .
$$

By Lemma 1.1, $\mathfrak{R} / J$ is pseudo-zero since $p$ and $q$ are co-prime. We have

$$
\begin{aligned}
|(\mathfrak{R} / J) \otimes \beta| & \sim\left|(\mathfrak{R} / J) \otimes \mathbb{Z}\left[A_{\Gamma}\right]\right| & & \text { by Proposition } 2.1(\mathrm{i}) \\
& \sim 1 & & \text { by Proposition } 1.3 .
\end{aligned}
$$

This completes the proof of the lemma.

2.7. Complexification of tor modules. Recall that $\mathfrak{R}_{\mathbb{C}}=\mathbb{C}\left[t_{1}^{ \pm 1}, \ldots, t_{n}^{ \pm 1}\right]$. Observe that

$$
\mathfrak{R}_{\mathbb{C}} \cong \mathfrak{R} \otimes_{\mathbb{Z}} \mathbb{C}, \text { and } \mathfrak{R}_{\mathbb{C}} \cong \mathfrak{R} \otimes_{\mathfrak{R}} \mathfrak{R}_{\mathbb{C}} .
$$

Let $I_{\mathbb{C}}$ be the $\mathbb{C}$-span of $I$ in $\mathfrak{R}_{\mathbb{C}}$. Then $I_{\mathbb{C}}$ is also the extension of $I$ from $\mathfrak{R}$ to $\mathfrak{R}_{\mathbb{C}}$.

Lemma 2.5. As $\mathbb{Z}$-modules, for every $i$,

$$
\left(\operatorname{Tor}_{i}^{\mathfrak{R}}(\Re / I, \beta)\right) \otimes_{\mathbb{Z}} \mathbb{C} \cong \operatorname{Tor}_{i}^{\Re_{\mathbb{C}}}\left(\Re_{\mathbb{C}} / I_{\mathbb{C}}, \beta_{\mathbb{C}}\right) .
$$

Proof. Since $\mathbb{C}$ is flat over $\mathbb{Z}$, we have $(\mathfrak{R} / I) \otimes_{\mathbb{Z}} \mathbb{C} \cong \mathfrak{R}_{\mathbb{C}} / I_{\mathbb{C}}$.

Since $\mathfrak{R}_{\mathbb{C}}$ is flat over $\mathfrak{R}$, we have $(\mathfrak{R} / I) \otimes_{\mathfrak{R}} \mathfrak{R} \mathbb{C} \cong \mathfrak{R}_{\mathbb{C}} / I_{\mathbb{C}}$. It follows that

$$
(\mathfrak{R} / I) \otimes_{\mathbb{Z}} \mathbb{C} \cong(\mathfrak{R} / I) \otimes_{\mathfrak{R}} \mathfrak{R}_{\mathbb{C}} .
$$

Suppose $\mathcal{C} \rightarrow \beta$ is a free resolution of $\beta$. By definition,

$$
\operatorname{Tor}_{i}^{\mathfrak{R}}(\mathfrak{R} / I, \beta)=H_{i}\left(\mathcal{C} \otimes_{\mathfrak{R}}(\mathfrak{R} / I)\right) .
$$

Tensoring (29) with $\mathfrak{R}_{\mathbb{C}}$, a flat $\mathfrak{R}$-module, we get

$$
\operatorname{Tor}_{i}^{\Re_{\mathbb{C}}}\left(\mathfrak{R}_{\mathbb{C}} / I_{\mathbb{C}}, \beta_{\mathbb{C}}\right)=H_{i}\left(\mathcal{C} \otimes_{\mathfrak{R}}(\mathfrak{R} / I) \otimes_{\mathfrak{R}} \mathfrak{R}_{\mathbb{C}}\right) .
$$

Tensoring (29) over $\mathbb{Z}$ with $\mathbb{C}$, a flat $\mathbb{Z}$-module, we get

$$
\left(\operatorname{Tor}_{i}^{\Re}(\Re / I, \beta)\right) \otimes_{\mathbb{Z}} \mathbb{C} \cong H_{i}\left(\left(\mathcal{C} \otimes_{\mathfrak{R}}(\Re / I)\right) \otimes_{\mathbb{Z}} \mathbb{C}\right) .
$$


Since $\mathcal{C}$ is free, each term of $\mathcal{C}$ is a direct of several $\mathfrak{R}$. It follows from (28) that the right hand sides of (30) and (31) are isomorphic as $\mathbb{Z}$-modules, whence the lemma.

\subsection{Proof of Proposition 2.1 (ii).}

Proof. a) The case $i=0$. Recall that $\operatorname{Tor}_{0}^{\mathfrak{R}}(\mathfrak{R} / I, \beta(p ; \Gamma))=(\mathfrak{R} / I) \otimes \beta$.

Since $(p) \subset I$, we have a natural surjection $\mathfrak{R} /(p) \rightarrow \mathfrak{R} / I$. Tensoring with $\beta=\beta(p ; \Gamma)$ we get a surjective map

$$
\beta \otimes(\mathfrak{R} /(p)) \rightarrow \beta \otimes(\mathfrak{R} / I) .
$$

Now $\beta \otimes(\Re /(p)) \cong \beta / p$, which is finite since $p$ acts on the finite-rank free abelian group $\beta$ by an injection, see Lemma 2.2(b). It follows that $\beta \otimes \mathfrak{R} / I$ is finite.

Since $\mathfrak{R} / I$ is pseudo-zero, by Proposition 2.1(i) and Proposition 1.3

$$
\left|\beta \otimes_{\mathfrak{R}}(\mathfrak{R} / I)\right| \sim\left|\mathbb{Z}\left[A_{\Gamma}\right] \otimes_{\mathfrak{R}}(\mathfrak{R} / I)\right| \sim 1 .
$$

b) The case $i=1$. First we show that $\operatorname{Tor}_{1}^{\mathfrak{R}}(\beta, \mathfrak{R} / I)$ is finite.

By Lemma 2.2(a), the $\mathfrak{R}_{\mathbb{C}}$-ideal generated by $\tilde{\alpha}_{\mathbb{C}}$ and $p$ is $\mathfrak{R}_{\mathbb{C}}$, hence $\tilde{\alpha}_{\mathbb{C}}+I_{\mathbb{C}}=\mathfrak{R}_{\mathbb{C}}$ because $p \in I$. It is well-known then (see eg. $\underline{\mathrm{AM}}$, Chapter 1])

$$
\tilde{\alpha}_{\mathbb{C}} \cap I_{\mathbb{C}}=\tilde{\alpha}_{\mathbb{C}} I_{\mathbb{C}}
$$

For two ideals $I_{1}, I_{2}$ in a commutative ring $R$, it is known that $\operatorname{Tor}_{1}^{R}\left(R / I_{1}, R / I_{2}\right) \cong I_{1} \cap I_{2} / I_{1} I_{2}$. Hence from (32) we have

$$
\operatorname{Tor}_{1}^{\mathfrak{R}_{\mathbb{C}}}\left(\mathfrak{R}_{\mathbb{C}} / \tilde{\alpha}_{\mathbb{C}}, \mathfrak{R}_{\mathbb{C}} / I_{\mathbb{C}}\right)=0 .
$$

Since $\beta_{\mathbb{C}}=\mathfrak{R}_{\mathbb{C}} / \tilde{\alpha}_{\mathbb{C}}$, this can be rewritten as

$$
\operatorname{Tor}_{1}^{\mathfrak{R}_{\mathbb{C}}}\left(\beta_{\mathbb{C}}, \mathfrak{R}_{\mathbb{C}} / I_{\mathbb{C}}\right)=0,
$$

which, by Lemma 2.5 implies that

$$
\left(\operatorname{Tor}_{1}^{\Re}(\beta, \Re / I)\right) \otimes_{\mathbb{Z}} \mathbb{C}=0 .
$$

Since $\operatorname{Tor}_{1}^{\mathfrak{R}}(\beta, \mathfrak{R} / I)$ is a finitely generated abelian group, (33) is equivalent to the $\operatorname{fact}^{\operatorname{Tor}_{1}^{\mathfrak{R}}}(\beta, \mathfrak{R} / I)$ is finite.

Now we show that $\left|\operatorname{Tor}_{1}^{\mathfrak{R}}(\beta, \mathfrak{R} / I)\right| \sim 1$. Since $\mathfrak{R} / I$ is pseudo-zero, $I \neq(p)$. This means there is $q \in I$ such that $q$ is not divisible by $p$. Since $p$ is irreducible, $p$ and $q$ are co-prime. Let $J=(p, q)$. Then $(p) \subset J \subset I$.

Tensoring $\beta$ with the exact sequence

$$
0 \rightarrow I / J \rightarrow \mathfrak{R} / J \rightarrow \mathfrak{R} / I \rightarrow 0
$$

we get the exact sequence

$$
\cdots \rightarrow \operatorname{Tor}_{1}^{\mathfrak{R}}(\mathfrak{R} / J, \beta) \rightarrow \operatorname{Tor}_{1}^{\mathfrak{R}}(\mathfrak{R} / I, \beta) \rightarrow((I / J) \otimes \beta) \rightarrow \ldots
$$

The module $I / J$, being a submodule of the pseudo-zero module $\mathfrak{R} / J$, is also pseudo-zero. Hence by Proposition 2.1(i) and Proposition 1.3.

$$
\left|\mathfrak{t o r}_{\mathbb{Z}}((I / J) \otimes \beta)\right| \sim 1 .
$$

By Lemma 2.4, $\operatorname{Tor}_{1}^{\mathfrak{R}}(\mathfrak{R} / J, \beta)$ is finite and has negligible growth,

$$
\left|\operatorname{Tor}_{1}^{\mathfrak{R}}(\mathfrak{R} / J, \beta)\right| \sim 1 .
$$

The middle term of (34), being finite, must satisfy

$$
\left|\operatorname{Tor}_{1}^{\Re}(\Re / I, \beta)\right| \leq\left|\operatorname{Tor}_{1}^{\Re}(\Re / J, \beta)\right|\left|\mathfrak{t o r}_{\mathbb{Z}}((I / J) \otimes \beta)\right|
$$

and hence by (34) and (36) is negligible, $\left|\operatorname{Tor}_{1}^{\mathfrak{R}}(\mathfrak{R} / I, \beta)\right| \sim 1$. 


\section{Proof of Theorems 6, 3, and 4]}

\subsection{Pseudo-zero kernel.}

Lemma 3.1. Suppose $M_{1}, M_{2}$ are finitely-generated $\mathfrak{R}$-module, $I \subset \mathfrak{R}$ is a prime ideal such that $\mathfrak{R} / I$ is pseudo-zero, and

$$
0 \rightarrow \mathfrak{R} / I \rightarrow M_{1} \rightarrow M_{2} \rightarrow 0
$$

is exact. Then $M_{1} \sim M_{2}$.

Proof. Choose a non-zero irreducible $p \in I$ and let $\beta=\beta(p ; \Gamma)$. Tensoring (37) with $\beta$, we get the exact sequence

$$
\cdots \rightarrow(\Re / I) \otimes \beta \rightarrow M_{1} \otimes \beta \rightarrow M_{2} \otimes \beta \rightarrow 0 .
$$

By Proposition 2.1(ii), ( $\Re / I) \otimes \beta$ is finite and $|(\mathfrak{R} / I) \otimes \beta| \sim 1$.

Applying Lemma 1.9 to the sequence (38), we get

$$
\left|\mathfrak{t o r}_{\mathbb{Z}}\left(M_{1} \otimes \beta\right)\right| \sim\left|\mathfrak{t o r}_{\mathbb{Z}}\left(M_{2} \otimes \beta\right)\right| .
$$

By Proposition $2.1(\mathrm{i}),\left|\mathfrak{t o r}_{\mathbb{Z}}\left(M_{i} \otimes \beta\right)\right| \sim\left|\mathfrak{t o r}_{\mathbb{Z}}\left(M_{i} \otimes \mathbb{Z}\left[A_{\Gamma}\right]\right)\right|$. Hence we can conclude that

$$
\mid \mathfrak{t o r}_{\mathbb{Z}}\left(M _ { 1 } \otimes \mathbb { Z } [ A _ { \Gamma } ] | \sim | \mathfrak { t o r } _ { \mathbb { Z } } \left(M_{2} \otimes \mathbb{Z}\left[A_{\Gamma}\right] \mid\right.\right. \text {. }
$$

This means $M_{1} \sim M_{2}$.

Lemma 3.2. Suppose $N, M_{1}$ and $M_{2}$ are finitely generated $\mathfrak{R}$-modules, and $N$ is pseudo-zero. If

$$
0 \rightarrow N \rightarrow M_{1} \rightarrow M_{2} \rightarrow 0
$$

is exact, then $M_{1} \sim M_{2}$.

Proof. It is well-known that there is a composition series

$$
N=N_{s} \supset N_{s-1} \supset \cdots \supset N_{1} \supset N_{0}=0
$$

such that for each $i, N_{i+1} / N_{i} \cong \Re / I_{i}$ for some prime ideal $I_{i}$, see eg. [Bou, Theorem IV.4.1]. We use induction on $s$. The case $s=1$ has been proved, see Lemma 3.1

Let $M_{1}^{\prime}=M_{1} / N_{s-1}$ and $N^{\prime}:=N / N_{s-1} \cong \mathfrak{R} / I$, with $I=I_{s-1}$. From (39) we have

$$
0 \rightarrow N^{\prime} \rightarrow M_{1}^{\prime} \rightarrow M_{2} \rightarrow 0 \text {. }
$$

From $M_{1}^{\prime}=M_{1} / N_{s-1}$, we have

$$
0 \rightarrow N_{s-1} \rightarrow M_{1} \rightarrow M_{1}^{\prime} \rightarrow 0 .
$$

Note that $N^{\prime}$ and $N_{s-1}$, being either a quotient or a submodule of the pseudo-zero module $N$, are pseudo-zero. By induction and the case $s=1$, from the exact sequences (41) and (42), we have

Hence $M_{1} \sim M_{2}$.

$$
M_{1}^{\prime} \sim M_{2}, \quad M_{1} \sim M_{1}^{\prime} .
$$

\subsection{Pseudo-zero quotient.}

Lemma 3.3. Suppose $N, M_{1}$ and $M_{2}$ are finitely generated $\mathfrak{R}$-modules, and $N$ is pseudo-zero. If

$$
0 \rightarrow M_{1} \rightarrow M_{2} \rightarrow N \rightarrow 0
$$

is exact, then $M_{1} \sim M_{2}$.

Proof. Again using induction on the length of the composition series (40) like in the proof of Lemma 3.2 we can assume that $N=\mathfrak{R} / I$, where $I \neq \mathfrak{R}$ is a prime module. Choose a non-zero irreducible $p \in I$ and let $\beta=\beta(p ; \Gamma)$.

Tensoring (43) with $\beta$, we have

$$
\cdots \rightarrow \operatorname{Tor}_{1}^{\mathfrak{R}}(\mathfrak{R} / I, \beta) \rightarrow M_{1} \otimes \beta \rightarrow M_{2} \otimes \beta \rightarrow \operatorname{Tor}_{0}^{\mathfrak{R}}(\mathfrak{R} / I, \beta) \rightarrow 0 .
$$

By Proposition 2.1(ii), $\operatorname{Tor}_{i}^{\Re}(\Re / I, \beta)$ is finite and $\left|\operatorname{Tor}_{i}^{\Re}(\Re / J, \beta)\right| \sim 1$ for $i=0,1$.

Applying Lemma 1.9 to the sequence (40), we get

$$
\left|\mathfrak{t o r}_{\mathbb{Z}}\left(M_{1} \otimes \beta\right)\right| \sim\left|\mathfrak{t o r}_{\mathbb{Z}}\left(M_{2} \otimes \beta\right)\right| \text {. }
$$


Using Proposition 2.1(i), we get

$$
\mid \mathfrak{t o r}_{\mathbb{Z}}\left(M _ { 1 } \otimes \mathbb { Z } [ A _ { \Gamma } ] | \sim | \mathfrak { t o r } _ { \mathbb { Z } } \left(M_{2} \otimes \mathbb{Z}\left[A_{\Gamma}\right] \mid,\right.\right.
$$

which means $M_{1} \sim M_{2}$.

\subsection{Proof of Theorem 6.}

Proof. Since $M_{1}$ and $M_{2}$ are pseudo-isomorphic, there are pseudo-zero $N_{1}$ and $N_{2}$ such that

$$
0 \rightarrow N_{1} \rightarrow M_{1} \rightarrow M_{2} \rightarrow N_{2} \rightarrow 0
$$

is exact. Then we have the following exact sequences

$$
\begin{aligned}
& 0 \rightarrow M_{1} / N_{1} \rightarrow M_{2} \rightarrow N_{2} \rightarrow 0 \\
& 0 \rightarrow N_{1} \rightarrow M_{1} \rightarrow M_{1} / N_{1} \rightarrow 0
\end{aligned}
$$

From (45) and Lemma 3.3 we have $M_{1} / N_{1} \sim M_{2}$, while from (46) and Lemma 3.2 we have $M_{1} / N_{1} \sim M_{1}$. It follows that $M_{1} \sim M_{2}$, which is equivalent to the statement of Theorem 6 ,

\subsection{The case when $M$ is torsion-free.}

Proposition 3.4. Suppose $M$ is a torsion-free finitely generated $\mathfrak{R}$-module. Then

$$
\lim _{\langle\Gamma\rangle \rightarrow \infty} \frac{\log \left|\operatorname{tor}_{\mathbb{Z}}\left(M \otimes \mathbb{Z}\left[\mathbb{Z}^{n} / \Gamma\right]\right)\right|}{\left|\mathbb{Z}^{n} / \Gamma\right|}=0
$$

We first prove the following lemma.

Lemma 3.5. Suppose $N, M_{1}$ and $M_{2}$ are finitely generated $\mathfrak{R}$-modules and

$$
0 \rightarrow M_{1} \rightarrow M_{2} \rightarrow N \rightarrow 0
$$

is exact. If $M_{2} \sim 0$, then $M_{1} \sim 0$.

Proof. Using induction on the length of a composition series (40) of $N$ we can assume that $N=$ $\mathfrak{R} / I$, where $I \subset \mathfrak{R}$ is a prime ideal.

If $\mathfrak{R} / I$ is pseudo-zero, then by Lemma 3.3. $M_{1} \sim M_{2} \sim 0$.

We will consider the remaining case, when $\mathfrak{R} / I$ is not pseudo-zero. Then $I$ is principal, $I=(p)$, where $p \in \mathfrak{R}$.

If $p=0$, then $N=\mathfrak{R}$ is free, and the sequence (47) is split, $M_{2} \cong M_{1} \oplus \mathfrak{R}$. One clearly has $\operatorname{tor}_{\mathbb{Z}}\left(M_{1} \otimes \mathbb{Z}\left[A_{\Gamma}\right]\right)=\operatorname{tor}_{\mathbb{Z}}\left(M_{2} \otimes \mathbb{Z}\left[A_{\Gamma}\right]\right)$, and the statement follows.

Suppose now $p \neq 0$. Let $\beta=\beta(p ; \Gamma)$. Tensoring (47) with $\beta$, the following is exact

$$
\cdots \rightarrow \operatorname{Tor}_{1}^{\Re}(\Re /(p), \beta) \rightarrow M_{1} \otimes \beta \rightarrow M_{2} \otimes \beta \rightarrow \Re /(p) \otimes \beta \rightarrow 0 .
$$

By Lemma 2.2(b), the first term is 0 . It follows that $M_{1} \otimes \beta$ is a subgroup of $M_{2} \otimes \beta$, and hence

$$
\left|\mathfrak{t o r}_{\mathbb{Z}}\left(M_{1} \otimes \beta\right)\right| \leq\left|\mathfrak{t o r}_{\mathbb{Z}}\left(M_{2} \otimes \beta\right)\right| .
$$

By Proposition 2.1(i),

$$
\left|\mathfrak{t o r}_{\mathbb{Z}}\left(M_{i} \otimes \beta\right)\right| \sim\left|\mathfrak{t o r}_{\mathbb{Z}}\left(M_{i} \otimes \mathbb{Z}\left[A_{\Gamma}\right]\right)\right|,
$$

and since $\left|\mathfrak{t o r}_{\mathbb{Z}}\left(M_{2} \otimes \mathbb{Z}\left[A_{\Gamma}\right]\right)\right| \sim 1$, we can conclude that $\left|\mathfrak{t o r}_{\mathbb{Z}}\left(M_{1} \otimes \mathbb{Z}\left[A_{\Gamma}\right]\right)\right| \sim 1$, or $M_{1} \sim 0$.

Proof of Proposition 3.4. Since $M$ is torsion free, the canonical map $M \rightarrow V:=M \otimes_{\mathfrak{R}} \mathcal{F}$, where $\mathcal{F}$ is the fractional field of $\mathfrak{R}$, is an embedding. This means $M$ is a lattice of $V$ with respect to $\mathfrak{R}$, and hence there is a free $\mathfrak{R}$-module $F$ such that $M$ embeds into $F$, see [Bou, Chepter 7]. One has an exact sequence of finitely-generated $\mathfrak{R}$-modules

$$
0 \rightarrow M \rightarrow F \rightarrow N \rightarrow 0 .
$$

We have $F \sim 0$ since $F$ is a free $\mathfrak{R}$-module. From Lemma 3.5 we conclude that $M \sim 0$. 


\subsection{Proof of Theorem 3 ,}

Proof. By Theorem 1.2, $M$ is and $\operatorname{tor}(M) \oplus M / \operatorname{tor}(M)$ are pseudo-isomorphic. Hence by Theorem 6 .

$$
M \sim(\operatorname{tor}(M) \oplus M / \operatorname{tor}(M))) .
$$

Since $M / \operatorname{tor}(M)$ is torsion-free, by Theorem 3.4 $M / \operatorname{tor}(M) \sim 0$. Hence we have

$$
M \sim \operatorname{tor}(M),
$$

The proof is thus reduced to the case when $M$ is a torsion module, which had been proved by $\mathrm{K}$. Schmidt, see [Sch, Theorem 21.1].

\subsection{Proof of Theorem 4,}

Proof. Suppose $\mathcal{D}$ is a chain complex of free finitely generated modules over a domain $R$,

$$
\cdots \rightarrow R^{m_{i+1}} \stackrel{\partial_{i+1}}{\longrightarrow} R^{m_{i}} \stackrel{\partial_{i}}{\longrightarrow} \ldots
$$

For our application either $R=\mathfrak{R}$ or $R=\mathbb{Z}$.

In the exact sequence

$$
0 \rightarrow\left(\operatorname{ker} \partial_{i} / \operatorname{Im} \partial_{i+1}\right) \rightarrow\left(R^{m_{i}} / \operatorname{Im} \partial_{i+1}\right) \rightarrow\left(R^{m_{i}} / \operatorname{ker} \partial_{i}\right) \rightarrow 0
$$

the first module is $H_{i}(\mathcal{D})$, the second coker $\partial_{i+1}$. Since the third is a torsion free $R$-module, one has

$$
\operatorname{tor}_{R}\left(H_{i}(\mathcal{D})\right)=\mathfrak{t o r}_{R}\left(\operatorname{coker} \partial_{i+1}\right) .
$$

Suppose now $\mathcal{C}$ is a chain complex of free finitely generated $\mathfrak{R}$-modules of the form

$$
\cdots \rightarrow \mathfrak{R}^{m_{i+1}} \stackrel{\partial_{i+1}}{\longrightarrow} \mathfrak{R}^{m_{i}} \stackrel{\partial_{i}}{\longrightarrow} \ldots
$$

Apply (49) to the above chain complex, we have

$$
\mathfrak{t o r}\left(H_{i}(\mathcal{C})\right)=\mathfrak{t o r}(M),
$$

where $M=\operatorname{coker} \partial_{i+1}$ which has a presentation

$$
\mathfrak{R}^{m_{i+1}} \stackrel{\partial_{i+1}}{\longrightarrow} \mathfrak{R}^{m_{i}} \rightarrow M \rightarrow 0 .
$$

Tensoring (51) with $\mathbb{Z}[A]$, where $A=\mathbb{Z}^{n} / \Gamma$, we get the exact sequence

$$
\mathbb{Z}[A]^{m_{i+1}} \stackrel{\partial_{i+1, \Gamma}}{\longrightarrow} \mathbb{Z}[A]^{m_{i}} \rightarrow M \otimes \mathbb{Z}[A] \rightarrow 0,
$$

from which it follows that

$$
M \otimes \mathbb{Z}[A]=\operatorname{coker}\left(\partial_{i+1, \Gamma}\right) .
$$

The complex $\mathcal{C} \otimes \mathbb{Z}[A]$ is

$$
\cdots \rightarrow \mathbb{Z}[A]^{m_{i+1}} \stackrel{\partial_{i+1}, \Gamma}{\longrightarrow} \mathbb{Z}[A]^{m_{i}} \stackrel{\partial_{i, \Gamma}}{\longrightarrow} \ldots
$$

Apply (49) to the chain complex (53), considered as complex over $\mathbb{Z}$, we get

$$
\mathfrak{t o r}_{\mathbb{Z}}\left(H_{i}(\mathcal{C} \otimes \mathbb{Z}[A])\right)=\mathfrak{t o r}_{\mathbb{Z}}\left(\operatorname{coker} \partial_{i+1, \Gamma}\right) .
$$

which, with (52), gives

$$
\mathfrak{t o r}_{\mathbb{Z}}\left(H_{i}(\mathcal{C} \otimes \mathbb{Z}[A])\right)=\mathfrak{t o r}_{\mathbb{Z}}(M \otimes \mathbb{Z}[A]) .
$$

Theorem 3, with identity (54), gives

$$
\limsup _{\langle\Gamma\rangle \rightarrow \infty} \frac{\log \left|\operatorname{tor}_{\mathbb{Z}}\left(H_{i}(\mathcal{C} \otimes \mathbb{Z}[A])\right)\right|}{\left|\mathbb{Z}^{n} / \Gamma\right|}=\mathbb{M}(\Delta(\operatorname{tor}(M)),
$$


From which and (50) we have

$$
\limsup _{\langle\Gamma\rangle \rightarrow \infty} \frac{\log \left|\mathfrak{t o r}_{\mathbb{Z}}\left(H_{i}(\mathcal{C} \otimes \mathbb{Z}[A])\right)\right|}{\left|\mathbb{Z}^{n} / \Gamma\right|}=\mathbb{M}\left(\Delta\left(\mathfrak{t o r}\left(H_{i}(\mathcal{C})\right)\right),\right.
$$

which completes the proof of Theorem 4 .

\section{Homology of abelian covering}

4.1. Alexander polynomials of links. Suppose $Z$ is an oriented integral homology 3 -sphere, i.e. $H_{i}(Z, \mathbb{Z}) \cong H_{i}\left(S^{3}, \mathbb{Z}\right)$, and $L \subset Z$ is an oriented link with $n$ ordered components. Let $N(L)$ be a small open tubular neighborhood of $L$ and $X=Z \backslash N(L)$. By Alexander duality $H_{1}(X, \mathbb{Z}) \cong \mathbb{Z}^{n}$, and there is a natural identification of $H_{1}(X, \mathbb{Z})$ with $\mathbb{Z}^{n}$ such that $t_{i}$ corresponds to the meridian of the the $i$-th component of the link. We fix such an identification of $H_{1}(X, \mathbb{Z})$ with $\mathbb{Z}^{n}$.

Let $\tilde{X}$ be the abelian covering corresponding to the abelianization $\pi_{1}(X) \rightarrow H_{1}(X, \mathbb{Z})=\mathbb{Z}^{n}$. The homology groups $H_{i}(\tilde{X}, \mathbb{Z})$ has a structure of $\mathfrak{R}=\mathbb{Z}\left[\mathbb{Z}^{n}\right]$ module. The Alexander polynomials $\Delta_{i}(L)$ (or $\Delta_{i}(L \subset Z)$ ), by definition, are the polynomials $\Delta_{i}\left(H_{1}(\tilde{X}, \mathbb{Z})\right)$. Recall that if $j$ is the smallest index such that $\Delta_{j}\left(H_{1}(\tilde{X}, \mathbb{Z})\right) \neq 0$, then one defines $\Delta\left(H_{1}(\tilde{X}, \mathbb{Z})\right)=\Delta_{j}\left(H_{1}(\tilde{X}, \mathbb{Z})\right)$. We also define $\Delta(L)=\Delta\left(H_{1}(\tilde{X}, \mathbb{Z})\right)$.

Note that $X$ has Euler characteristic 0 . It is known that $X$ is homotopic to a finite 2-dimensional CW-complex $Y$, with 10 -cell, $m+11$-cell $a_{1}, \ldots a_{m+1}$ and $m 2$-cell $b_{1}, \ldots, b_{m}$, for some number $m$. Certainly $m \geq n$. Let $\rho: \pi_{1}(Y) \rightarrow H_{1}(Y, \mathbb{Z})$ be the standard abelianization map. By choosing an appropriate CW-structure, we can assume further that $\rho\left(a_{i}\right)=t_{i}$ for $i=1, \ldots, n$.

Let $\tilde{Y}$ be the abelian covering of $Y$ corresponding to the abelianization $\rho: \pi_{1}(Y) \rightarrow H_{1}(Y, \mathbb{Z})=$ $\mathbb{Z}^{n}$. The CW-complex of $\tilde{Y}$ can be considered as a chain complex over $\mathfrak{R}=\mathbb{Z}\left[t_{1}^{ \pm 1}, \ldots, t_{n}^{ \pm 1}\right]$, and has the form

$$
0 \rightarrow \mathfrak{R}^{m} \stackrel{\partial_{2}}{\longrightarrow} \mathfrak{R}^{m+1} \stackrel{\partial_{1}}{\longrightarrow} \mathfrak{R} \rightarrow 0 .
$$

Here

$$
\partial_{1}=\left(\begin{array}{c}
1-\rho\left(a_{1}\right) \\
1-\rho\left(a_{2}\right) \\
\cdots \\
1-\rho\left(a_{m+1}\right)
\end{array}\right) .
$$

and $\partial_{2}$ is an $m \times(m+1)$-matrix with entries in $\Re$ which can be calculated using Fox derivative. There is only one 0 -cell of $\tilde{Y}$, denoted by $O$. The lift of $a_{i}$ beginning at $O$ will be denoted by $\tilde{a}_{i}$, $i=1, \ldots, m+1$.

Remark 4.1. The module $M_{2}=\operatorname{coker}\left(\partial_{2}\right)$ is known as the Alexander module. In some text eg [Hi], the Alexander polynomials are defined as $\Delta_{i}\left(M_{2}\right)$, which differ from ours only by a shift of index: $\Delta_{i}\left(H_{1}(\tilde{X}, \mathbb{Z})\right)=\Delta_{i+1}\left(M_{2}\right)$ since both $H_{1}(\tilde{X}, \mathbb{Z})$ and $M_{2}$ have the same $\mathfrak{R}$-torsion, see (49). In particular, $\Delta(L)=\Delta\left(H_{1}(\tilde{X}, \mathbb{Z})\right)=\Delta\left(M_{2}\right)$.

4.2. Homology of the branched covering. Suppose $\Gamma \subset \mathbb{Z}^{n}$ is a subgroup of finite index, and $A=A_{\Gamma}=\mathbb{Z}^{n} / \Gamma$. Let $X_{\Gamma}$ and $Y_{\Gamma}$ be the covering of $X$ and $Y$ respectively corresponding to the epimorphism $\pi_{1} \rightarrow H_{1} \rightarrow A$. Then the CW complex of $Y_{\Gamma}$ is $\mathcal{C}(\tilde{Y}) \otimes_{\mathfrak{R}} \mathbb{Z}[A]$ :

$$
0 \rightarrow \mathbb{Z}[A]^{m} \stackrel{\partial_{2, \Gamma}}{\longrightarrow} \mathbb{Z}[A]^{m+1} \stackrel{\partial_{1, \Gamma}}{\longrightarrow} \mathbb{Z}[A] \rightarrow 0
$$

The branched covering $X_{\Gamma}^{\mathrm{br}}$, by definition, is obtained from $X_{\Gamma}$ by Dehn fillings as follows. The boundary of $X$ is the union of $n$ tori, each surrounding a link component. The boundary of $X_{\Gamma}$ is also the union of several tori, each is the covering of one of tori in the boundary of $X$. Suppose $T$ is a torus in the boundary of $X_{\Gamma}$ covering the $i$-th torus of the boundary of $X$. There is a simple closed curve $C$ on $T$ covering the meridian of the $i$-th torus. To every boundary component $T$ one does the Dehn filling on $T$ that kills the homology class of $C$. The resulting 3-manifold is $X_{\Gamma}^{\text {br }}$.

The homology group $H_{1}\left(X_{\Gamma}^{\mathrm{br}}, \mathbb{Z}\right)$ is the the quotient of $H_{1}\left(X_{\Gamma}, \mathbb{Z}\right)$ by the relation $C=0$, for all the curves $C$ described in the above Dehn filling operation. The difficulty with working $H_{1}\left(X_{\Gamma}^{\mathrm{br}}, \mathbb{Z}\right)$ 
is these relations $C=0$ are local, they cannot be obtained from a global relation in terms of $\mathfrak{R}-$ modules.

We now describe a universal $\mathfrak{R}$-module through which the $\mathbb{Z}$-torsion of $H_{1}\left(X_{\Gamma}^{\mathrm{br}}, \mathbb{Z}\right)$ can be calculated.

Recall that $\partial_{2}$ in (55) is an $m \times m+1$ matrix. Let $I_{n, m+1}$ be the $n \times(m+1)$ matrix obtained from the identity $n \times n$ matrix by adding $(m+1-n)$ columns of 0 , and $T$ be the $n \times n$ diagonal matrix

$$
T=\operatorname{diag}\left(1-t_{1}, \ldots, 1-t_{n}\right) .
$$

Proposition 4.1. Let $M$ be the $\mathfrak{R}$-module with the following presentation matrix

$$
\left(\begin{array}{cc}
\partial_{2} & 0 \\
I_{n, m+1} & T
\end{array}\right),
$$

which has size $(m+n) \times(m+1+n)$. Then for any subgroup $\Gamma \subset \mathbb{Z}^{n}$ of finite index we have

$$
\operatorname{tor}_{\mathbb{Z}}\left(H_{1}\left(X_{\Gamma}^{\mathrm{br}}, \mathbb{Z}\right)\right) \cong \mathfrak{t o r}_{\mathbb{Z}}\left(M \otimes_{\mathfrak{R}} \mathbb{Z}\left[A_{\Gamma}\right]\right) .
$$

Proof. For $i=1, \ldots, n$ let $d_{i}=d_{i}(\Gamma)$ be the degree of $t_{i}$ in $A=\mathbb{Z}^{n} / \Gamma$, and $u_{i}=\sum_{l=0}^{d_{i}-1}\left(t_{i}\right)^{l} \in \mathbb{Z}[A]$.

The homology of the branched covering $H_{1}\left(X_{\Gamma}^{\mathrm{br}}, \mathbb{Z}\right)$ is

$$
H_{1}\left(X_{\Gamma}^{\mathrm{br}}, \mathbb{Z}\right)=H_{1}\left(Y_{\Gamma}, \mathbb{Z}\right) /(\mathrm{Rel}),
$$

where $(R e l)$ is the $\mathbb{Z}[A]$-submodule of $H_{1}\left(Y_{\Gamma}, \mathbb{Z}\right)$ generated by $u_{i} \tilde{a}_{i}, i=1, \ldots, n$.

By definition, $H_{1}\left(Y_{\Gamma}, \mathbb{Z}\right)$ is $H_{1}$ of the complex (56). By adding relations $u_{i} \tilde{a}_{i}=0, i=1, \ldots, n$, we see that $H_{1}\left(X_{\Gamma}^{\mathrm{br}}, \mathbb{Z}\right)$ is $H_{1}$ of the following complex

$$
0 \rightarrow \mathbb{Z}[A]^{m} \times \mathbb{Z}[A]^{n} \stackrel{D_{1}}{\longrightarrow} \mathbb{Z}[A]^{m+1} \stackrel{\partial_{1, \Gamma}}{\longrightarrow} \mathbb{Z}[A] \rightarrow 0
$$

where $D_{1}(x, y)=\partial_{2, \Gamma}(x)+U^{\prime}(y)$, with $U^{\prime}: \mathbb{Z}[A]^{n} \rightarrow \mathbb{Z}[A]^{m+1}$ being the $\mathbb{Z}[A]$-linear map defined by

$$
U^{\prime}\left(x_{1}, \ldots, x_{n}\right)=\left(u_{1} x_{1}, \ldots, u_{n} x_{n}, 0, \ldots, 0\right) .
$$

Let $U: \mathbb{Z}[A]^{n} \rightarrow \mathbb{Z}[A]^{n}$ be the $\mathbb{Z}[A]$-linear map given by the diagonal matrix $U=\operatorname{diag}\left(u_{1}, \ldots, u_{n}\right)$. Certainly $\operatorname{ker} U=\operatorname{ker} U^{\prime}$.

Applying (49) to the chain complex (57), we get

$$
\operatorname{tor}_{\mathbb{Z}}\left(H_{1}\left(X_{\Gamma}^{\mathrm{br}}, \mathbb{Z}\right)\right) \cong \mathfrak{t o r}_{\mathbb{Z}} \operatorname{coker} D_{1}
$$

The map $U^{\prime}: \mathbb{Z}[A]^{n} \rightarrow \mathbb{Z}[A]^{m+1}$ descends to $U^{\prime \prime}:\left(\mathbb{Z}[A]^{n} / \operatorname{ker} U^{\prime}\right) \rightarrow \mathbb{Z}[A]^{m+1}$, hence coker $D_{1}=$ coker $D_{1}^{\prime}$, where

$$
D_{1}^{\prime}: \mathbb{Z}[A]^{m} \times\left(\mathbb{Z}[A]^{n} / \operatorname{ker} U\right) \rightarrow \mathbb{Z}[A]^{m+1},
$$

defined by $D_{1}^{\prime}(x, y)=\partial_{2}(x)+U^{\prime \prime}(y)$. From (58) we have

$$
\operatorname{tor}_{\mathbb{Z}}\left(H_{1}\left(X_{\Gamma}^{\mathrm{br}}, \mathbb{Z}\right)\right) \cong \mathfrak{t o r}_{\mathbb{Z}}\left(\operatorname{coker} D_{1}^{\prime}\right)
$$

By tensoring $T: \mathfrak{R}^{n} \rightarrow \mathfrak{R}^{n}$ with $\mathbb{Z}[A]$, we get $T_{\Gamma}: \mathbb{Z}[A]^{n} \rightarrow \mathbb{Z}[A]^{n}$, which is given by a diagonal matrix. Note that $T_{\Gamma} U=0$, i.e. $T_{\Gamma}$ is 0 on the image of $U$, hence $T_{\Gamma}$ descend to a map $T_{\Gamma}^{\prime}:\left(\mathbb{Z}[A]^{n} / \operatorname{Im} U\right) \rightarrow \mathbb{Z}[A]^{n}$.

We have the following commutative diagram with exact vertical lines

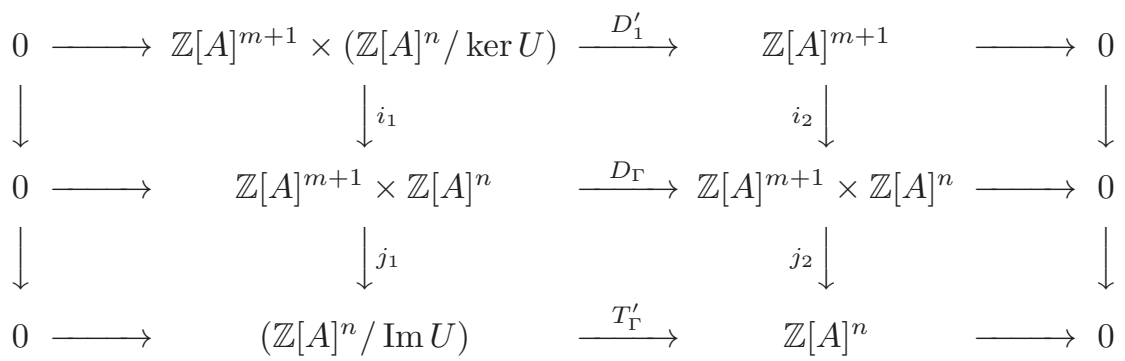

where $i_{1}(x, y)=(x, U(y)), i_{2}(x)=(x, 0), j_{1}(x, y)=(0, y), j_{2}(x, y)=y$, and $D_{\Gamma}$ is the matrix of presentation of $M$, tensoring with $\mathbb{Z}[A]$.

Let the first complex be $\mathcal{D}_{1}$, the second $\mathcal{D}_{2}$, and the 3 -rd $\mathcal{D}_{3}$. From the exact sequence $0 \rightarrow$ $\mathcal{D}_{1} \rightarrow \mathcal{D}_{2} \rightarrow \mathcal{D}_{3} \rightarrow 0$ we have a long exact sequence 


$$
\ldots H_{1}\left(\mathcal{D}_{3}\right) \rightarrow H_{0}\left(\mathcal{D}_{1}\right) \rightarrow H_{0}\left(\mathcal{D}_{2}\right) \rightarrow H_{0}\left(\mathcal{D}_{3}\right) \rightarrow 0 .
$$

The first term is 0 and the last term is free abelian group, by Lemma 4.2 below. Hence the second term and the third term in (60) have the same $\mathbb{Z}$-torsion. Since $H_{0}\left(\mathcal{D}_{1}\right)=$ coker $D_{1}^{\prime}$ and $H_{0}\left(\mathcal{D}_{2}\right)=M \otimes \mathbb{Z}[A]$, we have

$$
\operatorname{tor}_{\mathbb{Z}}\left(\operatorname{coker} D_{1}^{\prime}\right)=\operatorname{tor}_{\mathbb{Z}}(M \otimes \mathbb{Z}[A]),
$$

which, together with (59), proves the proposition.

Lemma 4.2. For chain complex $\mathcal{D}_{3}$

$$
0 \rightarrow\left(\mathbb{Z}[A]^{n} / \operatorname{Im} U\right) \stackrel{T_{\Gamma}^{\prime}}{\longrightarrow} \mathbb{Z}[A]^{n} \rightarrow 0
$$

one has $H_{1}(\mathcal{D})=0$ and $H_{0}(\mathcal{D})$ is a free abelian group.

Proof. This is the same as to show that for the chain $\mathcal{D}^{\prime}$

$$
0 \rightarrow \mathbb{Z}[A]^{n} \stackrel{U}{\longrightarrow} \mathbb{Z}[A]^{n} \stackrel{T_{\Gamma}}{\longrightarrow} \mathbb{Z}[A]^{n} \rightarrow 0
$$

one has $H_{1}\left(\mathcal{D}^{\prime}\right)=0$, and $H_{0}\left(\mathcal{D}^{\prime}\right)$ is free abelian.

Since both $U$ and $T_{\Gamma}$ are diagonal, $\mathcal{D}^{\prime}=\bigoplus_{i=1}^{n} \mathcal{D}_{i}^{\prime}$, where $\mathcal{D}_{i}^{\prime}$ is the complex

$$
0 \rightarrow \mathbb{Z}[A] \stackrel{u_{i}}{\longrightarrow} \mathbb{Z}[A]^{n} \stackrel{1-t_{i}}{\longrightarrow} \mathbb{Z}[A] \rightarrow 0 .
$$

As seen in subsection 1.7 the principal ideals $\left(u_{i}\right)$ and $\left(1-t_{i}\right)$ are annihilator of each other, hence $H_{1}\left(\mathcal{D}_{i}^{\prime}\right)=0$. Besides, the ideal $\left(1-t_{i}\right)$ is primitive as a lattice in $\mathbb{Z}[A]$, hence $H_{0}\left(\mathcal{D}_{i}^{\prime}\right)$ is a free abelian group.

\subsection{Proof of Theorem 2,}

Proof. Part (a), the case of non-branched covering, follows immediately from Theorem 5 .

Let us consider the case of branched covering. By Proposition 4.1, we have

$$
\begin{aligned}
\limsup _{\langle\Gamma\rangle \rightarrow \infty} \frac{\log \left|\mathfrak{t o r}_{\mathbb{Z}}\left(H_{1}\left(X_{\Gamma}^{\mathrm{br}}, \mathbb{Z}\right)\right)\right|}{\left|\mathbb{Z}^{n} / \Gamma\right|} & =\limsup _{\langle\Gamma\rangle \rightarrow \infty} \frac{\log \mathfrak{t o r}_{\mathbb{Z}}\left(M \otimes \mathbb{Z}\left[A_{\Gamma}\right]\right)}{\left|\mathbb{Z}^{n} / \Gamma\right|} \\
& =\mathbb{M}(\Delta(M)) \quad \text { by Theorem 3. }
\end{aligned}
$$

The module $M_{1}=\mathfrak{R}^{n} / T\left(\mathfrak{R}^{n}\right)$ has a free resolution

$$
0 \rightarrow \mathfrak{R}^{n} \stackrel{T}{\longrightarrow} \mathfrak{R}^{n} \rightarrow M_{1} \rightarrow 0,
$$

hence its projective dimension is 1 . Also $\Delta_{0}\left(M_{1}\right)=\prod_{i=1}^{n}\left(1-t_{i}\right)$.

Let $M_{2}=\operatorname{coker} \partial_{2}$. From the matrix of presentation of $M$ we see that there is an exact sequence

$$
0 \rightarrow M_{2} \rightarrow M \rightarrow M_{1} \rightarrow 0 .
$$

Since the projective dimension of $M_{1}$ is $\leq 1$, by [Hil, Theorem 3.12], one has

$$
\begin{aligned}
\Delta_{j}(M) & =\Delta_{j}\left(M_{2}\right) \Delta_{0}\left(M_{1}\right) \\
& =\Delta_{j}\left(M_{2}\right) \prod_{i=1}^{n}\left(1-t_{i}\right) .
\end{aligned}
$$

It follows that $\Delta(M)=\Delta\left(M_{2}\right) \prod_{i=1}^{n}\left(1-t_{i}\right)$. Since $\mathbb{M}\left(1-t_{i}\right)=1$ and $\Delta\left(M_{2}\right)=\Delta(L)$, we have

$$
\mathbb{M}(\Delta(M))=\mathbb{M}(\Delta(L)),
$$

from which and (61) one gets part (b) of Theorem 2 


\section{CONVERGING SEQUENCES}

5.1. Statement. For a non-zero vector $x \in \mathbb{R}^{n}$ let $\mathbf{r}(x)=x /\|x\| \in S^{n-1}$ be the unit vector positively colinear with $x$. Here $S^{n-1}$ is the $(n-1)$-dimensional sphere of unit vectors in $\mathbb{R}^{n}$. For a subgroup $\Gamma \subset \mathbb{Z}^{n}$ of finite index let $d_{i}=d_{i}(\Gamma)$ be the degree of $t_{i}$ in the quotient group $A_{\Gamma}=\mathbb{Z}^{n} / \Gamma$. Let $\mathbf{r}(\Gamma)=\mathbf{r}\left(d_{1}, \ldots, d_{n}\right) \in S_{+}^{n-1}$, the part of $S^{n-1}$ with non-negative coordinates.

Theorem 7. Suppose $M$ is a finitely-generated $\mathfrak{R}$-module. For any $\kappa \in S_{+}^{n-1}$, there exists a sequence of finite index subgroups $\Gamma_{s} \subset \mathbb{Z}^{n}, s=1,2, \ldots$ such that

$$
\lim _{s \rightarrow \infty} \mathbf{r}\left(\Gamma_{s}\right)=\kappa
$$

and

$$
\limsup _{s \infty} \frac{\log \left|\operatorname{tor}_{\mathbb{Z}}\left(M \otimes \mathbb{Z}\left[\mathbb{Z}^{n} / \Gamma_{s}\right]\right)\right|}{\left|\mathbb{Z}^{n} / \Gamma_{s}\right|}=\mathbb{M}(\Delta(M)) .
$$

Remark 5.1. One could prove a similar statement, replacing $M \otimes \mathbb{Z}\left[\mathbb{Z}^{n} / \Gamma_{s}\right]$ with $H_{i}\left(\mathcal{C} \otimes \mathbb{Z}\left[\mathbb{Z}^{n} / \Gamma_{s}\right]\right)$ like in Theorem 4

The proof and methods of this section are independent of Theorem 3 . It gives an alternative proof of "half" of Theorem 3. The left hand side in the identity of Theorem 3 is greater than or equal to the right hand side.

5.2. A result of Bombieri and Zannier: reduction from $\mathbb{Z}^{n}$ to $\mathbb{Z}$. For $\mathbf{k} \in \mathbb{Z}^{n}$ let $\mathbf{k}^{\perp}=$ $\left\{\mathbf{m} \in \mathbb{Z}^{n} \mid \mathbf{k} \cdot \mathbf{m}=0\right\}$, where $\mathbf{k} \cdot \mathbf{m}$ is the usual dot product. Define

$$
\langle\mathbf{k}\rangle=\left\langle\mathbf{k}^{\perp}\right\rangle=\min \left\{|x|, x \in \mathbf{k}^{\perp} \backslash\{0\}\right\} .
$$

The group homomorphism $\mathbb{Z}^{n} \rightarrow \mathbb{Z}$ given by $\mathbf{m} \rightarrow \mathbf{m} \cdot \mathbf{k}$ gives rise to the algebra homomorphism $\tau_{\mathbf{k}}: \mathbb{Q}\left[t_{1}^{ \pm 1}, \ldots, t_{n}^{ \pm 1}\right] \rightarrow \mathbb{Q}\left[t^{ \pm 1}\right]$ defined by

$$
\tau_{\mathbf{k}}\left(t^{\mathbf{m}}\right)=t^{\mathbf{m} \cdot \mathbf{k}}
$$

The following is a deep result of Bombieri and Zannier [SZ, BMZ, which was formulated as a conjecture by Schinzel.

Theorem 5.1. Suppose $p_{1}, p_{2} \in \mathbb{Q}\left[t_{1}^{ \pm 1}, \ldots, t_{n}^{ \pm 1}\right]$ are co-prime. There is a constant $C=C\left(p_{1}, p_{2}\right)$ such that if $\langle\mathbf{k}\rangle>C$, then $\operatorname{gcd}\left(\tau_{\mathbf{k}}\left(p_{1}\right), \tau_{\mathbf{k}}\left(p_{2}\right)\right)$ is the product of some (possibly none) cyclotomic polynomials.

From this one can easily deduce the following.

Proposition 5.2. Suppose $p_{1}, \ldots, p_{\ell} \in \mathbb{Z}\left[t_{1}^{ \pm 1}, \ldots, t_{n}^{ \pm 1}\right]$. There exists a constant $C=C\left(p_{1}, \ldots, p_{k}\right)$ such that if $\langle\mathbf{k}\rangle>C$, then

$$
\operatorname{gcd}\left(\tau_{\mathbf{k}}\left(p_{1}\right), \ldots, \tau_{\mathbf{k}}\left(p_{\ell}\right)\right)=\phi \tau_{\mathbf{k}}\left(\operatorname{gcd}\left(p_{1}, \ldots, p_{\ell}\right)\right),
$$

where $\phi$ is a product of cyclotomic polynomials.

Proof. By dividing each of $p_{i}$ by $\operatorname{gcd}\left(p_{1}, \ldots, p_{\ell}\right)$ we can assume that $\operatorname{gcd}\left(p_{1}, \ldots, p_{\ell}\right)=1$.

We will use induction on $\ell$. But first make the following well-known observation on the coefficients of $\tau_{\mathbf{k}}(p)$ of a polynomial $p \in \mathbb{Z}\left[t_{1}^{ \pm 1}, \ldots, t_{n}^{ \pm 1}\right]$ having the form

$$
p=\sum_{\mathbf{m} \in \mathcal{N}} c_{\mathbf{m}} t^{\mathbf{m}}
$$

where $\mathcal{N} \subset \mathbb{Z}$ is a finite set. Then

$$
\tau_{\mathbf{k}}(p)=\sum_{\mathbf{m} \in \mathcal{N}} c_{\mathbf{m}} t^{\mathbf{m} \cdot \mathbf{k}}
$$

If $\langle\mathbf{k}\rangle$ is greater than $\left|\mathbf{m}-\mathbf{m}^{\prime}\right|$ for any two $\mathbf{m} \neq \mathbf{m}^{\prime} \in \mathcal{N}$, then $\mathbf{k} \cdot \mathbf{m} \neq \mathbf{k} \cdot \mathbf{m}^{\prime}$, and (62) shows that the coefficients of $\tau_{\mathbf{k}}(p)$, in some order, are exactly the coefficients of $p$.

Now we proceed with induction. Suppose $\ell=2$. By Bombieri and Zannier result, over $\mathbb{Q}\left[t_{1}^{ \pm 1}, \ldots, t_{n}^{ \pm 1}\right], \operatorname{gcd}\left(\tau_{\mathbf{k}}\left(p_{1}\right), \tau_{\mathbf{k}}\left(p_{2}\right)\right)=\phi$, a product of cyclotomic polynomials. Hence over $\mathbb{Z}\left[t_{1}^{ \pm 1}, \ldots, t_{n}^{ \pm 1}\right]$, $\operatorname{gcd}\left(p_{1}, p_{2}\right)=a \phi$, for some integer $a$. It follows that $a$ is the gcd of all the coefficients of $\tau_{\mathbf{k}}\left(p_{1}\right)$ and $\tau_{\mathbf{k}}\left(p_{2}\right)$. By the above observation, with $\langle\mathbf{k}\rangle$ big enough, this means $a$ is the gcd of all the coefficients of $p_{1}$ and $p_{2}$, which must be 1 . This proves the statement when $\ell=2$. 
Now assume $\ell \geq 3$. One has

$$
\begin{aligned}
\operatorname{gcd}\left(\tau_{\mathbf{k}}\left(p_{1}\right), \ldots, \tau_{\mathbf{k}}\left(p_{\ell}\right)\right) & =\operatorname{gcd}\left(\tau_{\mathbf{k}}\left(p_{1}\right), \operatorname{gcd}\left(\tau_{\mathbf{k}}\left(p_{2}\right), \ldots, \tau_{\mathbf{k}}\left(p_{\ell}\right)\right)\right. \\
& =\operatorname{gcd}\left(\tau_{\mathbf{k}}\left(p_{1}\right), \phi \tau_{\mathbf{k}}\left(\operatorname{gcd}\left(p_{2}, \ldots, p_{\ell}\right)\right) \quad\right. \text { by induction } \\
& =\phi^{\prime} \tau_{\mathbf{k}}\left(\operatorname{gcd}\left(p_{1}, \operatorname{gcd}\left(p_{2}, \ldots, p_{\ell}\right)\right)\right) \quad \text { by case } \ell=2 \\
& =\phi^{\prime} \tau_{\mathbf{k}}\left(\operatorname{gcd}\left(p_{1}, p_{2}, \ldots, p_{\ell}\right)\right) .
\end{aligned}
$$

Here $\phi, \phi^{\prime}$ are product of cyclotomic polynomials. The proof is completed.

5.3. A result of Lawton. Recall that the additive Mahler measure $\mathbb{M}(f)$ of $f \in \mathbb{C}\left[t_{1}^{ \pm 1}, \ldots, t_{n}^{ \pm 1}\right], f \neq$ 0 , is defined by

$$
\mathbb{M}(f)=\int_{\mathbb{S}^{n}} \log |f(x)| d \sigma,
$$

where $\mathbb{S}^{n}$ is the $n$-dimensional torus, and $d \sigma$ is the invariant Haar measure on $\mathbb{S}^{n}$ normalized so that $\int_{\mathbb{S} n} d \sigma=1$.

The Mahler measure is additive, $\mathbb{M}(f g)=\mathbb{M}(f)+\mathbb{M}(g)$. It is known that $\mathbb{M}(f)=0$ is and only $f$ is a generalized cyclotomic polynomial, see e.g. SZ, Sch.

The following approximation result was a conjecture of D. Boyd, and was proved by Lawton, see e.g. [Law, SZ, Sch.

Theorem 5.3. (Lawton) Suppose $f \in \mathbb{C}\left[t_{1}^{ \pm 1}, \ldots, t_{n}^{ \pm 1}\right], f \neq 0$. Then

$$
\lim _{\langle\mathbf{k}\rangle \rightarrow \infty} \mathbb{M}\left(\tau_{\mathbf{k}}(f)\right)=\mathbb{M}(f) \text {. }
$$

5.4. A converging sequence. The following follows from Bombieri-Zannier and Lawton results. Denote $\Re_{1}=\mathbb{Z}\left[t^{ \pm 1}\right]$.

Proposition 5.4. Let $\mathbf{k}^{(s)} \in \mathbb{Z}^{n}, s=1,2, \ldots$ be any sequence such that $\lim _{s \rightarrow \infty}\left\langle\mathbf{k}^{(s)}\right\rangle=\infty$, and $M$ a finitely-generated $\mathfrak{R}$-module. Let $M^{(s)}=M \otimes \mathfrak{R}_{1}$, where $\mathfrak{R}_{1}$ is considered as an $\mathfrak{R}$-module via $\tau_{s}:=\tau_{\mathbf{k}^{(s)}}: \mathfrak{R} \rightarrow \mathfrak{R}_{1}$. Then

$$
\lim _{s \rightarrow \infty} \mathbb{M}\left(\Delta\left(M^{(s)}\right)\right)=\mathbb{M}(\Delta(M))
$$

Remark 5.2. It not true in general that $\lim _{s \rightarrow \infty} \Delta\left(M^{(s)}\right)=\Delta(M)$.

Proof. Suppose $M$ has a presentation matrix $\partial$ of size $m_{1} \times m_{0}$, with entries in $\mathfrak{R}$. Then $M^{(s)}$ has presentation matrix $\tau_{s}(\partial)$, with entries in $\mathfrak{R}_{1}$.

Let $j$ be the smallest integer such that $\Delta_{j}(M) \neq 0$. This means all the $\left(m_{0}-j^{\prime}\right)$-minor of $\partial$ is 0 if $j^{\prime}<j$, and if the $\left(m_{0}-j\right)$-minors of $\partial$ are $p_{1}, \ldots, p_{\ell} \in \Re$, then

$$
\Delta(M)=\Delta_{j}(M)=\operatorname{gcd}\left(p_{1}, \ldots, p_{\ell}\right) .
$$

Note that every minor of $\tau_{s}(\partial)$ is obtained from the corresponding minor by by applying $\tau_{s}$. It follows that all the $\left(m_{0}-j^{\prime}\right)$-minor of $\tau_{s}(\partial)$ is 0 if $j^{\prime}<j$, and the $m_{0}-j$ minors are $\tau_{s}\left(p_{1}\right), \ldots, \tau_{s}\left(p_{\ell}\right)$.

By Proposition 5.2 , for $s$ big enough,

$$
\operatorname{gcd}\left(\tau_{s}\left(f_{1}\right), \ldots, \tau_{s}\left(f_{r}\right)\right)=\phi \tau_{s}\left(\operatorname{gcd}\left(f_{1}, \ldots, f_{r}\right)\right),
$$

where $\phi$ is a product of cyclotomic polynomials. This means

$$
\Delta\left(M^{(s)}\right)=\phi \tau_{s}(\Delta(M)) .
$$

Using additivity of the Mahler measure and the fact that the Mahler measure of a cyclotomic polynomial is 0 , we have

$$
\mathbb{M}\left(\Delta\left(M^{(s)}\right)\right)=\mathbb{M}\left(\tau_{s}(\Delta(M))\right) \text {. }
$$

Since $\left\langle\mathbf{k}^{(s)}\right\rangle \rightarrow \infty$ as $s \rightarrow \infty$, by Lawton theorem, we have

$$
\lim _{s \rightarrow \infty} \mathbb{M}\left(\Delta\left(M^{(s)}\right)\right)=\lim _{s \rightarrow \infty} \mathbb{M} \tau_{s}(\Delta(M))=\mathbb{M}(\Delta(M)) .
$$


5.5. Theorem $\left[3\right.$, the case $n=1$. In the previous section we approximate $\mathbb{Z}^{n}$ by $\mathbb{Z}^{n} /\left(\mathbf{k}^{\perp}\right)$, which has rank 1. Now we want to approximate abelian rank 1 group by finite cyclic group. Here we give a short, independent of previous sections, proof of the case $n=1$ of Theorem 3 .

Proposition 5.5. Suppose $M$ is a finitely generated $\mathfrak{R}_{1}$-module. Then

$$
\lim _{\ell \rightarrow \infty} \frac{\operatorname{tor}_{\mathbb{Z}}(M \otimes \mathbb{Z}[\mathbb{Z} / \ell])}{\ell}=\mathbb{M}(\Delta(\operatorname{tor} M)) .
$$

Proof. The reason the case $n=1$ is easy is $\mathbb{Z}[\mathbb{Z} / \ell]=\mathfrak{R}_{1} /\left(1-t^{\ell}\right)$, with $\left(1-t^{\ell}\right)$ a principal ideal.

For an $\mathfrak{R}_{1}$-module $N$ and an element $a \in \mathfrak{R}_{1}$ let ${ }_{a} N$ be the $a$-torsion of $N$ :

$$
{ }_{a} N=\{x \in N \mid a x=0\} .
$$

A homological interpretation of ${ }_{a} N$ is $\operatorname{Tor}_{1}^{\mathfrak{R}_{1}}\left(N, \mathfrak{R}_{1} /(a)\right)={ }_{a} N$. If $a \mid b$ then ${ }_{a} N \subset{ }_{b} N$. If $N$ is a finitely-generated torsion module, then there is $b \in \mathfrak{R}_{1}$, called a universal annihilator of $N$, such that for every $a \in \mathfrak{R}_{1}$,

$$
{ }_{a} N=\operatorname{gcd}(b, a) N .
$$

For example, such $b$ can be the product of all the generators of all prime ideals associated to $N$. One can also define $b$ as the least common multiple of the family of annihilators of a generating family for $N$.

Since $M^{\prime}=M / \operatorname{tor}(M)$ is torsion free, by [Bou, Chapter VII], there is a free $\mathfrak{R}_{1}$-module $F$ such that $M^{\prime} \subset F$ and $F / M^{\prime}$ is a torsion module. Let $f$ be a universal annihilator of $F / M^{\prime}$. Decompose $f=f_{1} f_{2}$, where $f_{1}$ is the product of all cyclotomic factors (with multiplicity) in the prime decomposition of $f$. The identity map $F \rightarrow F$ descends to a surjective map

$$
F /\left(f_{1} F\right) \rightarrow f_{1}\left(F / M^{\prime}\right) .
$$

Since $f_{1}$ is monic, $F / f_{1} F$ is a finitely generated $\mathbb{Z}$-module. It follows that $f_{1}\left(F / M^{\prime}\right)$ is a finitely generated $\mathbb{Z}$-module, hence its $\mathbb{Z}$-torsion part is a finite set.

Tensoring the exact sequence

$$
0 \rightarrow M^{\prime} \rightarrow F \rightarrow F / M^{\prime} \rightarrow 0
$$

with $\mathbb{Z}[\mathbb{Z} / \ell]$, we get

$$
0 \rightarrow \operatorname{Tor}_{1}^{\Re_{1}}\left(F / M^{\prime}, \mathbb{Z}[\mathbb{Z} / \ell]\right) \rightarrow M^{\prime} \otimes \mathbb{Z}[\mathbb{Z} / \ell] \rightarrow F \otimes \mathbb{Z}[\mathbb{Z} / \ell] \rightarrow\left(F / M^{\prime}\right) \otimes \mathbb{Z}[\mathbb{Z} / \ell] \rightarrow 0 .
$$

Since $F$ is a free $\mathfrak{R}_{1}$-module, the third term is a free $\mathbb{Z}$-module. It follows that the $\mathbb{Z}$-torsions of the first and the second terms are the same

$$
\mathfrak{t o r}_{\mathbb{Z}}\left(\operatorname{Tor}_{1}^{\Re_{1}}\left(F / M^{\prime}, \mathbb{Z}[\mathbb{Z} / \ell]\right)\right)=\mathfrak{t o r}_{\mathbb{Z}}\left(M^{\prime} \otimes \mathbb{Z}[\mathbb{Z} / \ell]\right) .
$$

Note that

$$
\operatorname{Tor}_{1}^{\Re_{1}}\left(F / M^{\prime}, \mathbb{Z}[\mathbb{Z} / \ell]\right)={ }_{\left(1-t^{\ell}\right)}\left(F / M^{\prime}\right)
$$

is a subset of $f_{1}\left(F / M^{\prime}\right)$ since $1-t^{\ell}$ is a product of cyclotomic polynomial. Since $\left|\mathfrak{t o r}_{\mathbb{Z}}\left(f_{1}\left(F / M^{\prime}\right)\right)\right|$ is finite and does not depend on $\ell$, we conclude that $\mathfrak{t o r}_{\mathbb{Z}}\left(M^{\prime} \otimes \mathbb{Z}[\mathbb{Z} / \ell]\right)$ is finite and bounded from above.

Tensoring the exact sequence

$$
0 \rightarrow \operatorname{tor}(M) \rightarrow M \rightarrow M^{\prime} \rightarrow 0
$$

with $\mathbb{Z}[\mathbb{Z} / \ell]=\Re_{1} /\left(1-t^{\ell}\right)$, taking into account $\operatorname{Tor}_{1}^{\Re_{1}}\left(M^{\prime}, \Re_{1} /\left(1-t^{\ell}\right)=0\right.$, we have

$$
0 \rightarrow \operatorname{tor}(M) \otimes \mathbb{Z}[\mathbb{Z} / \ell] \rightarrow M \otimes \mathbb{Z}[\mathbb{Z} / \ell] \rightarrow M^{\prime} \otimes \mathbb{Z}[\mathbb{Z} / \ell] \rightarrow 0 .
$$

Since the $\mathbb{Z}$-torsion of the last term is bounded, we see that the $\mathbb{Z}$-torsion parts of the first two terms have the same growth, i.e. $M \sim \operatorname{tor}(M)$. The Proposition now follows from the case of torsion modules, which was known [Sch] (see also [GS, Ri, Lü2]). 


\subsection{Converging sequences.}

Lemma 5.6. Let $M$ be a finitely generated $\mathfrak{R}$-module, and $\mathbf{k}^{(s)} \in \mathbb{Z}^{n}, s=1,2, \ldots$ be any sequence such that $\lim _{s \rightarrow \infty}\left\langle\mathbf{k}^{(s)}\right\rangle=\infty$ and $\operatorname{gcd}\left(k_{1}^{(s)}, \ldots, k_{n}^{(s)}\right)=1$ for $s \geq 1$. For each positive integer $j$ define the subgroup $\Gamma_{s, j} \subset \mathbb{Z}^{n}$ by

$$
\Gamma_{s, j}=\left(\mathbf{k}^{(s)}\right)^{\perp}+j \mathbf{k}^{(s)} .
$$

For every $s$ there exists an integer $\eta_{s}>0$ such that for every sequence $j_{s}>\eta_{s}$, we have

$$
\lim _{s \rightarrow \infty} \frac{\log \left|\mathfrak{t o r}_{\mathbb{Z}}\left(M \otimes \mathbb{Z}\left[A_{\Gamma_{s, j_{s}}}\right]\right)\right|}{\left|\mathbb{Z}^{n} / \Gamma_{s, j_{s}}\right|}=\mathbb{M}(\Delta(M)) .
$$

Proof. It is easy to see that for any $\mathbf{k}=\left(k_{1}, \ldots, k_{n}\right) \in \mathbb{Z}^{n}$ with $\operatorname{gcd}\left(k_{1}, \ldots, k_{n}\right)=1$, and $0<j \in \mathbb{Z}$, the $\operatorname{map} \mathbf{m} \rightarrow \mathbf{m} \cdot \mathbf{k}(\bmod j)$ is an isomorphism between $\mathbb{Z}^{n} /\left(\mathbf{k}^{\perp} \oplus j \mathbf{k}\right)$ and $\mathbb{Z} /\left(j|\mathbf{k}|^{2}\right)$.

It follows that, as $\mathfrak{R}$-modules,

$$
M \otimes_{\mathfrak{R}} \mathbb{Z}\left[\mathbb{Z}^{n} / \Gamma_{s, j}\right] \cong M^{(s)} \otimes_{\mathfrak{R}_{1}} \mathbb{Z}\left[\mathbb{Z} /\left(j\left|\mathbf{k}^{(s)}\right|^{2}\right)\right] .
$$

Since $\left|\mathbb{Z}^{n} / \Gamma_{s, j}\right|=j\left|\mathbf{k}^{(s)}\right|^{2}$, one has

$$
\begin{aligned}
& \lim _{j \rightarrow \infty} \frac{\log \left|\mathfrak{t o r}_{\mathbb{Z}}\left(M \otimes \mathbb{Z}\left[A_{\Gamma_{s, j}}\right]\right)\right|}{\left|\mathbb{Z}^{n} / \Gamma_{s, j}\right|}=\lim _{j \rightarrow \infty} \frac{\left|\mathfrak{t o r}_{\mathbb{Z}}\left(M^{(s)} \otimes_{\mathfrak{R}_{1}} \mathbb{Z}\left[\mathbb{Z} /\left(j \| \mathbf{k}^{(s)}||^{2}\right)\right]\right)\right|}{j\left|\mathbf{k}^{(s)}\right|^{2}}, \\
& \lim _{j \rightarrow \infty} \frac{\log \left|\mathfrak{t o r}_{\mathbb{Z}}\left(M \otimes \mathbb{Z}\left[A_{\Gamma_{s, j}}\right]\right)\right|}{\left|\mathbb{Z}^{n} / \Gamma_{s, j}\right|}=\mathbb{M}\left(\Delta\left(M^{(s)}\right)\right) \quad \text { by Proposition 5.5. }
\end{aligned}
$$

Let $a_{s, j}$ be the left hand side of (63). From (63), for fixed $s$, there is $\eta_{s}>0$ such that if $j>\eta_{s}$, then

$$
\mid a_{s, j}-\mathbb{M}\left(\Delta\left(M^{(s)}\right) \mid<1 / s\right.
$$

It is clear if $j_{s}>\eta_{s}$, then

$$
\lim _{s \rightarrow \infty} a_{s, j_{s}}=\lim _{s \rightarrow \infty} \mathbb{M}\left(\Delta\left(M^{(s)}\right)\right)=\mathbb{M}(\Delta(M)),
$$

where the last identity follows from Proposition 5.4

5.7. Proof of Theorem [7. Assume the sequence $\mathbf{k}^{(s)}$ of Lemma 5.6 satisfies $k_{i}^{(s)} \neq 0$ for $i=$ $1, \ldots, n$. If we choose $j_{s}$ divisible by the product $k_{1}^{(s)} \ldots k_{n}^{(s)}$, then $d_{i}\left(\Gamma_{s, j_{s}}\right)=\left|j_{s} / k_{i}^{(s)}\right|$, and

$$
\mathbf{r}\left(\Gamma_{s, j_{s}}\right)=\mathbf{r}\left(1 /\left|k_{1}^{(s)}\right|, \ldots, 1 /\left|k_{n}^{(s)}\right|\right) .
$$

Thus Theorem 7 follows from Lemma 5.6 and the following.

Lemma 5.7. Suppose $\kappa \in S_{+}^{n-1}$. There exists $\mathbf{k}^{(s)}=\left(k_{1}^{(s)}, \ldots, k_{n}^{(s)}\right) \in \mathbb{Z}^{n}$ such that

(i) $k_{1}^{(s)}, \ldots, k_{n}^{(s)}>0$ and have greatest common divisor 1 .

(ii) $\lim _{s \rightarrow \infty} \mathbf{r}\left(1 / k_{1}^{(s)}, \ldots, 1 / k_{n}^{(s)}\right)=\kappa$.

(iii) $\lim _{s \rightarrow \infty}\left\langle\mathbf{k}^{(s)}\right\rangle=\infty$.

Proof. Let $S_{++}^{n-1}$ be the subset of $S^{n-1}$ consisting of points with all positive coordinates. Let Inv : $S_{++}^{n-1} \rightarrow S_{++}^{n-1}$ be the map defined by

$$
\operatorname{Inv}\left(x_{1}, \ldots, x_{n}\right)=\mathbf{r}\left(1 / x_{1}, \ldots, 1 / x_{n}\right) .
$$

It is clear that Inv is an involution, and hence is a auto-diffeomorphism of $S_{++}^{n-1}$.

The set $Q$ of all points of the form $\mathbf{r}\left(k_{1}, \ldots, k_{n}\right)$, with $k_{1}, \ldots, k_{n}$ positive and co-prime, is dense in $S_{+}^{n-1}$. If $\mathcal{L}$ is a finite collection of hyperplanes in $\mathbb{R}^{n}$, then $Q \backslash \mathcal{L}$ is still dense in $S_{+}^{n-1}$.

For $s \geq 1$ let $P_{s}$ be the set of all points in $\mathbb{Z}^{n}$ having norm $\leq s$, and

$$
\mathcal{L}_{s}=\bigcup_{s \in P_{s}} \mathbf{k}^{\perp} \text {. }
$$

By definition, if $\mathbf{k} \notin \mathcal{L}_{s}$, then $\langle\mathbf{k}\rangle>s$.

$Q \backslash \mathcal{L}_{s}$ is dense in $S_{+}^{n-1}$, hence so is $\operatorname{Inv}\left(Q \backslash \mathcal{L}_{s}\right)$. This implies there is $\mathbf{x}^{(s)} \in Q \backslash \mathcal{L}_{s}$ such that

$$
\left\|\operatorname{Inv}\left(\mathbf{k}^{(s)}\right)-\kappa\right\|<1 / s .
$$


By definition, $\mathbf{x}^{(s)}=\mathbf{r}\left(\mathbf{k}^{(s)}\right)$ for some $\mathbf{k}^{(s)}=\left(k_{1}^{(s)}, \ldots, k_{n}^{(s)}\right)$ with positive and co-prime $k_{i}^{(s)}$. Since $\mathbf{k}^{(s)} \notin \mathcal{L}_{s}$, we have $\left\langle\mathbf{k}^{(s)}\right\rangle>s$, which establishes Property (ii). Inequality (64) shows that Property (iii) also holds.

\section{REFERENCES}

[AM] M. Atiyah and I. Macdonald, Introduction to commutative algebra, Addison-Wesley Publishing Co., Reading, Mass.-London-Don Mills, Ont. 1969.

[BM] H. Bass and M. P. Murthy, Grothendieck groups and Picard groups of abelian group rings, Ann. of Math. (2), 86 (1967), 16-73.

[BV] N. Bergeron and A. Venkatesh, The asymptotic growth of torsion homology for arithmetic groups, Preprint (2010), arXiv:1004.1083

[Ber] D. Bertrand, Duality on tori and multiplicative dependence relations, J. Austral. Math. Soc. Ser. A 62 (1997), 198-216.

[BMZ] E. Bombieri, D. Masser, and U. Zannier, Anomalous subvarieties structure theorems and applications, Int. Math. Res. Not. IMRN 2007, no. 19, Art. ID rnm057, 33 pp.

[Bou] N. Bourbaki, Commutative algebra. Chapters 1-7, Elements of Mathematics (Berlin), Springer-Verlag, Berlin, 1989.

[EW] M. Einsiedler and T. Ward, Fitting ideals for finitely presented algebraic dynamical systems, Aequationes Math. 60 (2000), 57-71.

[GS] F. Gonzalez-Acuna and H. Short, Cyclic branched coverings of knots and homology spheres, Revista Math. 4 (1991), 97-120.

[Go] C. McA. Gordon, Knots whose branched coverings have periodic homology, Trans. Amer. Math. Soc. 168 (1972), 357-370.

[Hi] J. Hillman, Algebraic invariants of links, Series on Knots and Everything, 32, World Scientific Publishing Co., Inc., River Edge, NJ, 2002.

[HS] J. Hillman and M. Sakuma On the homology of finite abelian coverings of links, Canad. Math. Bull. 40 (1997), 309-315.

[Ka] I. Kaplansky, Commutative rings, The University of Chicago Press, Chicago, 1974.

[Lau] M. Laurent, Equations diophantiennes exponentielles, Invent. Math. 78 (1984), 299-327.

[Law] W. Lawton, A problem of Boyd concerning geometric means of polynomials J. Number Theory 16 (1983), 356-362.

[LSW] D. Lind, K. Schmidt, and T. Ward, Mahler measure and entropy for commuting automorphisms of compact groups, Invent. Math. 101 (1990), 593-629.

[Le1] T. Le, Hyperbolic volume, Mahler measure, and homology growth, talk at Columbia University (2009), slides available from http://www.math.columbia.edu/ volconf09/notes/leconf.pdf

[Le2] T. Le, Hyperbolic volume and homology growth, to appear.

[Lü1] W. Lück, Approximating $L^{2}$-invariants by their finite-dimensional analogues, Geom. Funct. Anal. 4 (1994), 455-481.

[Lü2] W. Lück, $L^{2}$-invariants: theory and applications to geometry and KK-theory, Ergebnisse der Mathematik und ihrer Grenzgebiete. 3. Folge. A Series of Modern Surveys in Mathematics, 44, Springer-Verlag, Berlin, 2002.

[Mü] W. Müller, Analytic torsion and cohomology of hyperbolic 3-manifolds, Preprint of the Max-Planck Institute, Bonn (2009).

$[\mathrm{MM}] \quad$ J. Mayberry and K. Murasugi, Torsion-groups of abelian coverings of links, Trans. Amer. Math. Soc. 271 (1982), 143-173.

[Ra] J. Raimbault, Exponential growth of torsion in abelian coverings, Preprint (2010).

[Ri] R. Riley, Growth of order of homology of cyclic branched covers of knots, Bull. London Math. Soc. 22 (1990), 287-297.

[SZ] A. Schinzel, Polynomials with special regard to reducibility. With an appendix by Umberto Zannier, Encyclopedia of Mathematics and its Applications, 77, Cambridge University Press, Cambridge, 2000.

[Sch] K. Schmidt, Dynamical systems of algebraic origin, Progress in Mathematics, 128 Birkhäuser Verlag, Basel, 1995.

[Sch-W] W. Schmidt, Heights of points on subvarieties of $G_{m}^{n}$, Number theory (Tiruchirapalli, 1996), 97-99, Contemp. Math., 210, Amer. Math. Soc., Providence, RI, 1998.

[SW] D. Silver and S. Williams, Mahler measure, links and homology growth, Topology 41 (2002), 979-991.

[Tu] V. Turaev, Introduction to combinatorial torsions, Birkhäuser, Basel, 2001.

Department of Mathematics, Georgia Institute of Technology, Atlanta, GA 30332-0160, USA

E-mail address: letu@math.gatech.edu 